\title{
Simulations of fiber flocculation: Effects of fiber properties and interfiber friction
}

\author{
Christian F. Schmid, Leonard H. Switzer, and Daniel J. Klingenberga) \\ Department of Chemical Engineering and Rheology Research Center, \\ University of Wisconsin, Madison, Wisconsin 53706
}

(Received 18 November 1999; final revision received 27 March 2000)

\section{Synopsis}

Non-Brownian fibers commonly flocculate in flowing suspensions at relatively low concentrations ( $<1 \%$ by weight). We have developed a particle-level simulation technique modeling fibers as chains of rods connected by hinges to probe fiber flocculation. The model incorporates fiber flexibility, irregular fiber equilibrium shapes, and frictional fiber interactions. Model fibers reproduce known orbits of isolated rigid and flexible fibers in shear flow. Simulation predictions of first normal stress differences in homogeneously dispersed, dilute flexible fiber suspensions agree with experimental data. Fiber features such as flexibility and irregular equilibrium shapes strongly impact single fiber and suspension behavior. Fibers aggregate in simulations with interfiber friction, in the absence of attractive forces between fibers. Strong flocculation is observed in suspensions of stiff fibers with irregular equilibrium shapes. Flocs contain many fibers with three or more contact points, and derive cohesiveness from elastic energy held in fibers-consistent with the elastic interlocking mechanism of flocculation. At higher concentrations $\left(n L^{3} \approx 100\right.$, where $n$ is the fiber number density and $L$ is the fiber length), coherent fiber networks form in simulations. Average numbers of contacts per fiber and contact force magnitudes in sheared and static networks are compared with existing fiber network theory predictions. (c) 2000 The Society of Rheology. [S0148-6055(00)01404-8]

\section{INTRODUCTION}

In applications such as papermaking and composites processing, fibrous particles are suspended in flowing viscous media. Predicting and controlling fiber motion in these processes is critical to achieving the desired spatial arrangements of fibers in products. A complication is that fibers in these suspensions do not always move affinely with the fluid, but rather aggregate [Mason (1950), Kerekes et al. (1985), Kerekes and Schell (1992)]. A major goal in the processing of fiber-filled fluids is to understand how fiber mass distribution in flowing suspensions is affected by suspension conditions, and microscopic fiber features and interaction forces. Direct observation of fiber motions in concentrated, flowing suspensions is difficult, as fibers are small, opaque, and moving rapidly in most applications. In this work, a particle-level simulation technique is developed to systematically probe microstructure and flocculation in flowing fiber suspensions.

Much is known about the motion of isolated fibers in low Reynolds number flow. Jeffery (1922) solved for the motion of an isolated prolate spheroid in simple shear flow, $v_{x}^{\infty}=\dot{\gamma} y$, showing that a spheroid rotates periodically, spending most of the time nearly

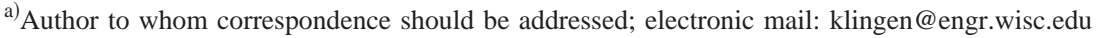


aligned with the flow. The spheroid rotation period $T$ increases with increasing spheroid aspect ratio $r_{s}=a / b$ (ratio of major to minor axis lengths) as $T=2 \pi\left(r_{s}+1 / r_{s}\right) / \dot{\gamma}$. Jeffery's analysis was shown to be valid for any axisymmetric body with fore-aft symmetry, provided that an equivalent aspect ratio $r_{e}$ is used in place of the actual spheroid aspect ratio $r_{s}$ [Bretherton (1962)]. For rigid cylinders of length-to-diameter ratio $r_{p}$ $=L / D$, equivalent aspect ratios have been experimentally measured [Goldsmith and Mason (1967)], yielding the correlation $r_{e}=1.24 r_{p} / \sqrt{\ln r_{p}}$ [Cox (1971)].

Forgacs and Mason (1959a) and Goldsmith and Mason (1967) have theoretically and experimentally investigated the flow induced deformation of single fibers in simple shear flow. A cylindrical fiber in the flow/gradient plane is predicted to bend when the dimensionless group (called the bending ratio BR here)

$$
\mathrm{BR} \equiv \frac{E_{Y}\left(\ln 2 r_{e}-1.50\right)}{(\mu \dot{\gamma}) 2 r_{p}^{4}}
$$

is less than one, where $E_{Y}$ is the fiber Young's modulus and $\mu$ is the fluid viscosity. Fiber shapes observed experimentally in shear flow have been classified and correlated with values of BR [Forgacs and Mason (1959b)]. Salinas and Pittman (1981) have quantified fiber bending by measuring the minimum radius of curvature $R_{\min }$ attained by fibers during their orbits, finding smaller $R_{\min }$ with decreasing BR.

Studies have elucidated the forces responsible for holding fibers in flocs. Mason $(1950,1954)$ was the first to recognize that flocs form under conditions where electromagnetic and chemical forces are negligible compared to hydrodynamic forces. He proposed that, above a critical volume fraction $\phi_{v, \text { crit }}=3 /\left(2 r_{p}^{2}\right)$, at which exactly one fiber center of mass (c.m.) is present in the spherical volume swept out by a fiber of length $L$, extensive fiber collisions result in mechanical fiber entanglement. Meyer and Wahren (1964) applied the concept of mechanical entanglement to highly concentrated fiber networks, suggesting that network strength derives from the interlocking of elastically loaded fibers. Assuming randomly oriented fibers, they derived an expression for the average number of contact points per fiber $\left\langle n_{c}\right\rangle$ in a network as a function of $r_{p}$ and $\phi_{v}$. They also predicted that the network shear modulus increases with increasing $r_{p}, \phi_{v}$, and fiber stiffness. This and similar models for fiber network yield stress [Bennington et al. (1990)] and individual floc strength [Farnood et al. (1994)] agree qualitatively with experiments, supporting the mechanical interlocking hypothesis.

Less is known about the processes by which fibers flocculate. Mason (1950) postulated that flocculation is a dynamic equilibrium process, with fibers continuously entering and leaving flocs, both rates being equal at steady state. Kerekes (1995) has identified dimensionless groups that help predict flocculation in specific experiments. He defines the crowding factor, $N_{c}=2 \phi_{v} r_{p}^{2} / 3$, which is the average number of fiber c.m.s present in the spherical volume $\pi L^{3} / 6$. A closely related quantity is $n L^{3}=6 N_{c} / \pi$, the number of fiber c.m.s per unit volume $n$ times the cubic volume $L^{3}$. Though useful, the crowding factor understandably cannot explain all phenomena, such as the dramatic dispersive action of water-soluble polymer in pulp fiber suspensions [Zauscher et al. (1999)]. Thus, a technique that explores the effects of microscopic fiber features and interaction forces on suspension behavior is needed.

Direct particle level simulations have been employed to study non-Brownian fiber suspension flow behavior. Rigid fiber simulations have incorporated long range hydrodynamic interactions (HI) [Mackaplow and Shaqfeh (1996), Harlen et al. (1999)], short range lubrication forces [Yamane et al. (1994)], and combined long and short range HI [Claeys and Brady (1993), Fan et al. (1998)]. Other rigid fiber simulations incorporate 


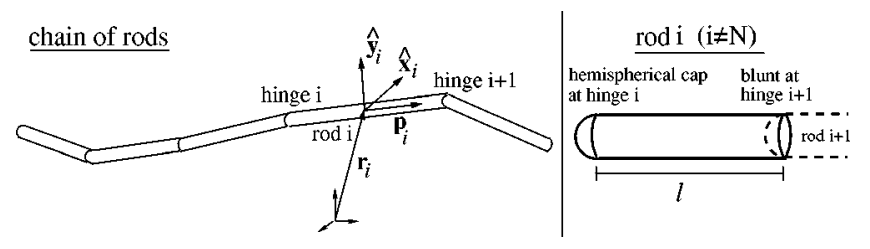

FIG. 1. Mechanical model of a fiber.

purely repulsive [Sundararajakumar and Koch (1997)], and repulsive plus frictional [Thomasset et al. (1997), Harlen et al. (1999)] contact forces. Sundararajakumar and Koch (1997) and Harlen et al. (1999) showed that hydrodynamic lubrication cannot prevent physical contact between fibers, and thus nonhydrodynamic contact forces will become important. Sundararajakumar and Koch (1997) show that for concentrated rigid fiber suspensions $\left(n L^{3}>40\right)$, simulations that neglect long range HI but include mechanical contacts predict fiber suspension dynamics (shear viscosity and orientation distributions) more accurately than results that include $\mathrm{HI}$ but neglect contacts. In simulations of falling ball rheometry, Harlen et al. (1999) showed that nonhydrodynamic contact forces dominate over hydrodynamic interactions as the concentration is increased $\left(n L^{3} \geq 12\right)$. Thus, in our study of fiber flocculation, we neglect hydrodynamic interactions and probe the effects of repulsive and frictional fiber/fiber interaction forces. Simulations of flexible fibers, composed of linked rigid spheres [Yamamoto and Matsuoka (1996), Skjetne et al. (1997)] and spheroids [Ross and Klingenberg (1997)] have successfully replicated single fiber motions and predicted suspension viscosities. Following Ross and Klingenberg (1997), we employ chains of elongated bodies (rigid rods), enabling the simulation of high aspect ratio $\left(r_{p}=50-280\right)$ fibers with relatively few bodies per chain.

In Sec. II, details of our simulation technique are presented. The model is shown to reproduce experimentally observed single fiber motion and homogeneous suspension rheological properties in Sec. III. Results of simulations probing fiber flocculation are then presented in Sec. IV, demonstrating that flocculation can be induced by purely mechanical means (interparticle friction, elastic fiber deformation, and irregular equilibrium shapes) - without attractive forces. Simulated flocs derive cohesiveness from elastic energy storage in fibers, consistent with the elastic interlocking mechanism of flocculation. Fiber features and interactions are shown to dramatically impact flocculation.

\section{SIMULATION METHOD}

\section{A. Mechanical model and equations of motion}

A fiber is modeled as a chain of $N$ rigid rods and $N-1$ hinges, as shown in Fig. 1. Rods have side length $l$, diameter $D$, and aspect ratio $r_{p_{r}} \equiv l / D$. The chain contour length is $L=N l$, and fiber aspect ratio $r_{p} \equiv L / D$. Rods rotate and twist about the hinges, replicating fiber bending and twisting deformations. However, the chain contour length remains fixed. Index $i$ refers to a rod, as well as the hinge preceding it, (hinge 1 is fictitious). Each rod $i$ in a chain (except for $\operatorname{rod} N$ ) has a hemispherical end cap at hinge $i$, and is blunt at hinge $i+1$. Rod $N$ has hemispherical caps on both ends. When a chain is straight, the hemispherical end of each rod $i$ overlaps the adjacent rod $i-1$. This model retains a smooth fiber surface even when bent.

A fiber configuration is specified by $3(N+1)$ independent coordinates; the fiber c.m. position $\boldsymbol{r}_{\text {c.m. }}$. relative to space-fixed coordinates, and $3 N$ internal coordinates relating the 
orientations of $N$ orthonormal sets of rod base vectors $\left(\hat{\boldsymbol{x}}_{i}, \hat{\boldsymbol{y}}_{i}, \boldsymbol{p}_{i}\right)$ (illustrated in Fig. 1) to the space-fixed frame. Rod c.m. positions are obtained by

$$
\begin{gathered}
\boldsymbol{r}_{1}=\boldsymbol{r}_{\mathrm{c} . \mathrm{m} .}-\frac{l}{2 N} \sum_{i=2}^{N}\left(\boldsymbol{p}_{1}+\boldsymbol{p}_{i}+2 \sum_{j=2}^{i-1} \boldsymbol{p}_{j}\right), \\
r_{i(\neq 1)}=\boldsymbol{r}_{1}+\frac{l}{2} \boldsymbol{p}_{1}+l \sum_{j=2}^{i-1} \boldsymbol{p}_{j}+\frac{l}{2} \boldsymbol{p}_{i} .
\end{gathered}
$$

All vectors are based in the space-fixed frame unless otherwise indicated by bracketed subscripts, e.g., $\boldsymbol{v}_{\text {[frame] }}$.

Equations of motion in Cartesian coordinates for chains of rigid rods connected by hinges are derived from force and torque balances on each rod in a chain, with the constraint that the fiber contour length remains constant. For certain fiber interactions, additional constraints are imposed on the motion of contacting fibers. Balances contain contributions from five different sources: hydrodynamic forces and torques, body forces, elastic bending and twisting torques, constraint forces and their moments (to hold rods together in a chain), and interparticle forces (repulsive and frictional) and their moments. Particle and fluid acceleration are neglected.

The force balance on rod $i$ is

$$
\boldsymbol{F}_{i}^{h}-\boldsymbol{X}_{i}+\boldsymbol{X}_{i+1}+\boldsymbol{F}_{i}^{b}+\sum_{j} \boldsymbol{f}_{i j}=\mathbf{0}
$$

where $\boldsymbol{F}_{i}^{h}$ is the hydrodynamic force, $\boldsymbol{X}_{i}$ and $\boldsymbol{X}_{i+1}$ are the constraint forces in hinges $i$ and $i+1$, respectively, $\boldsymbol{F}_{i}^{b}$ is the body force, and $\Sigma_{j} f_{i j}$ is the net interparticle force from all rods $j$ interacting with $\operatorname{rod} i$. The torque balance on $\operatorname{rod} i$ is

$$
\boldsymbol{T}_{i}^{h}+\left(-\frac{l}{2} \boldsymbol{p}_{i}\right) \times\left(-\boldsymbol{X}_{i}\right)+\frac{l}{2} \boldsymbol{p}_{i} \times \boldsymbol{X}_{i+1}-\boldsymbol{Y}_{i}+\boldsymbol{Y}_{i+1}+\sum_{j} \boldsymbol{t}_{i j}=\mathbf{0},
$$

where $\boldsymbol{T}_{i}^{h}$ is the hydrodynamic torque, the second and third terms are the moments about the rod $i$ c.m. exerted by the constraint forces acting in hinges $i$ and $i+1$, respectively, $\boldsymbol{Y}_{i}=\boldsymbol{Y}_{i}^{\mathrm{bend}}+\boldsymbol{Y}_{i}^{\mathrm{twist}}$ is the sum of the bending and twisting torques in hinge $i$, and $\boldsymbol{Y}_{i+1}$ is the analogous quantity for hinge $i+1$. The term $\Sigma_{j} \boldsymbol{t}_{i j}=\Sigma_{j}\left(S_{i j} \boldsymbol{p}_{i}+0.5 G_{i j} \boldsymbol{n}_{i j}\right) \times \boldsymbol{f}_{i j}$ is the net moment about the rod $i \mathrm{c} . \mathrm{m}$. exerted by the interparticle forces acting on $\operatorname{rod} i$, where $S_{i j}$ is the point on the rod $i$ centerline at which the interaction with rod $j$ occurs, the unit vector $\boldsymbol{n}_{i j}$ points from rod $i$ to rod $j$ at the interaction point and is perpendicular to both rod surfaces, and $G_{i j}$ is the separation distance between rod centerlines at the interaction point.

In this model, the suspending fluid is Newtonian and hydrodynamic interactions between rods in different fibers, as well as within the same fiber, are neglected. The hydrodynamic force on a rod $i$ is

$$
\boldsymbol{F}_{i}^{h}=\boldsymbol{A}_{i} \cdot\left(\boldsymbol{v}_{i}^{\infty}-\dot{\boldsymbol{r}}_{i}\right),
$$

where $\dot{\boldsymbol{r}}_{i}$ is the translational velocity of the $\operatorname{rod} i$ c.m., $\boldsymbol{v}_{i}^{\infty}$ is the ambient fluid velocity at the rod $i$ c.m., and $\boldsymbol{A}_{i}=3 \pi \mu l\left[Y^{A} \boldsymbol{\delta}+\left(X^{A}-Y^{A}\right) \boldsymbol{p}_{i} \boldsymbol{p}_{i}\right]$ is the second order, hydrodynamic resistance tensor. The resistance functions $X^{A}\left(r_{s}\right)$ and $Y^{A}\left(r_{s}\right)$ for a prolate spheroid of aspect ratio $r_{s}$ [Kim and Karrila (1991)] are used, with an effective aspect ratio $r_{e_{r}}$ in 
place of $r_{s}$. The choice of $r_{e_{r}}$ is explained in Sec. III. In this work, lengths are scaled with rod radius $R$ except where noted. For simulations of simple shear flow, $v_{x}^{\infty}=\dot{\gamma} y$, time is scaled with the inverse of the shear rate $1 / \dot{\gamma}$ (strain $\gamma \equiv \dot{\gamma} t$ ), and all forces with $3 \pi \mu \dot{\gamma} l R$, (superscripted asterisks denote dimensionless variables). The dimensionless rod velocity $\dot{\boldsymbol{r}}_{i}^{*} \equiv \dot{\boldsymbol{r}}_{i} / R \dot{\gamma}$, obtained from Eq. (3), is

$$
\dot{\boldsymbol{r}}_{i}^{*}=\boldsymbol{v}_{i}^{\infty *}+\boldsymbol{A}_{i}^{-1 *} \cdot\left(-\boldsymbol{X}_{i}^{*}+\boldsymbol{X}_{i+1}^{*}+\boldsymbol{F}_{i}^{b *}+\sum_{j} f_{i j}^{*}\right)
$$

where

$$
\boldsymbol{A}_{i}^{-1 *}=\left[\frac{1}{Y^{A}} \boldsymbol{\delta}+\left(\frac{1}{X^{A}}-\frac{1}{Y^{A}}\right) \boldsymbol{p}_{i} \boldsymbol{p}_{i}\right]
$$

The hydrodynamic torque on $\operatorname{rod} i$ is

$$
\boldsymbol{T}_{i}^{h}=\boldsymbol{C}_{i} \cdot\left(\boldsymbol{\Omega}_{i}^{\infty}-\boldsymbol{\omega}_{i}\right)+\tilde{\boldsymbol{H}}: \dot{\gamma}
$$

where $\boldsymbol{\omega}_{i}$ is the angular velocity of $\operatorname{rod} i, \boldsymbol{\Omega}_{i}^{\infty}$ is the ambient fluid angular velocity at the rod $i$ c.m., and $\dot{\gamma}=\frac{1}{2}\left(\nabla \boldsymbol{v}^{\infty}+\nabla \boldsymbol{v}^{\infty \dagger}\right)$ is the rate of deformation tensor of the ambient fluid. The resistance tensors $\boldsymbol{C}_{i}=\pi \mu l^{3}\left[Y^{C} \boldsymbol{\delta}+\left(X^{C}-Y^{C}\right) \boldsymbol{p}_{i} \boldsymbol{p}_{i}\right]$, and $\tilde{\boldsymbol{H}}$ $=-\pi \mu l^{3} Y^{H}\left(\boldsymbol{\epsilon} \cdot \boldsymbol{p}_{i}\right) \boldsymbol{p}_{i}$ [Kim and Karrila (1991)], where $\boldsymbol{\epsilon}$ is the third order permutation tensor, employ the resistance functions $X^{C}\left(r_{s}\right), Y^{C}\left(r_{s}\right)$, and $Y^{H}\left(r_{s}\right)$ for a prolate spheroid, with an effective aspect ratio $r_{e_{r}}$ used in place of $r_{s}$. From Eq. (7), a torque-free spheroid has the angular velocity given by Jeffery (1922),

$$
\boldsymbol{\omega}^{\mathrm{jeff}}=\mathbf{\Omega}^{\infty}-\boldsymbol{C}^{-1} \cdot \tilde{\boldsymbol{H}}: \dot{\gamma}
$$

For simple shear flow $v_{x}^{\infty}=\dot{\gamma} y$, the ambient fluid angular velocity is $\boldsymbol{\Omega}^{\infty}=(0,0$, $-0.5 \dot{\gamma})$, and $\boldsymbol{C}^{-1} \cdot \tilde{\boldsymbol{H}}: \dot{\boldsymbol{\gamma}}=-0.5 \dot{\gamma} Y^{H}\left(p_{x} p_{z},-p_{y} p_{z}, p_{y}^{2}-p_{x}^{2}\right) / Y^{C}$. The dimensionless rod angular velocity can thus be obtained from Eq. (4) as

$$
\boldsymbol{\omega}_{i}^{*}=\boldsymbol{\omega}_{i}^{\mathrm{jeff} *}+\boldsymbol{C}_{i}^{-1 *} \cdot\left(\frac{3}{4 r_{p_{r}}^{2}} \sum_{j} \boldsymbol{t}_{i j}^{*}-\boldsymbol{Y}_{i}^{*}+\boldsymbol{Y}_{i+1}^{*}\right)+\frac{3}{4 r_{p_{r}} Y^{C}} \boldsymbol{p}_{i} \times\left(\boldsymbol{X}_{i}^{*}+\boldsymbol{X}_{i+1}^{*}\right),
$$

where $t_{i j}^{*}=t_{i j} /\left(3 \pi \mu \dot{\gamma} l R^{2}\right), \boldsymbol{Y}_{i}^{*}=\boldsymbol{Y}_{i} /\left(\pi \mu \dot{\gamma} l^{3}\right)$, and

$$
\boldsymbol{C}_{i}^{-1 *}=\left[\frac{1}{Y^{C}} \boldsymbol{\delta}+\left(\frac{1}{X^{C}}-\frac{1}{Y^{C}}\right) \boldsymbol{p}_{i} \boldsymbol{p}_{i}\right]
$$

In this work, body forces are not applied. They are retained in the equations of motion for completeness.

Bending and twisting torques attempt to hold a chain in a specified equilibrium shape. Fiber equilibrium shape is defined by fixing a coordinate system $\left(\hat{\boldsymbol{x}}_{i}, \hat{\boldsymbol{y}}_{i}, \boldsymbol{p}_{i}\right)$ on each rod $i$, and an equilibrium coordinate system $\left(\hat{\boldsymbol{x}}_{i}^{\mathrm{eq}}, \hat{\boldsymbol{y}}_{i}^{\mathrm{eq}}, \boldsymbol{p}_{i}^{\mathrm{eq}}\right)$ for each rod $i$ on the preceding rod $i-1$ [Fig. 2(a)]. At equilibrium, the coordinate system fixed on rod $i$ and its equilibrium frame on rod $i-1$ are aligned. The relative orientation between the equilibrium orientation for rod $i\left(\hat{\boldsymbol{x}}_{i}^{\mathrm{eq}}, \hat{\boldsymbol{y}}_{i}^{\mathrm{eq}}, \boldsymbol{p}_{i}^{\mathrm{eq}}\right)$ and the orientation of $\operatorname{rod} i-1\left(\hat{\boldsymbol{x}}_{i-1}, \hat{\boldsymbol{y}}_{i-1}, \boldsymbol{p}_{i-1}\right)$ is prescribed. Vector $\hat{\boldsymbol{y}}_{i}^{\mathrm{eq}}$ is rotated by an angle $\phi^{\mathrm{eq}}$ relative to $\hat{\boldsymbol{y}}_{i-1}$, and vector $\boldsymbol{p}_{i}^{\mathrm{eq}}$ is rotated by an angle $\theta^{\text {eq }}$ relative to $\boldsymbol{p}_{i-1}$, as shown in Fig. 2(b). 

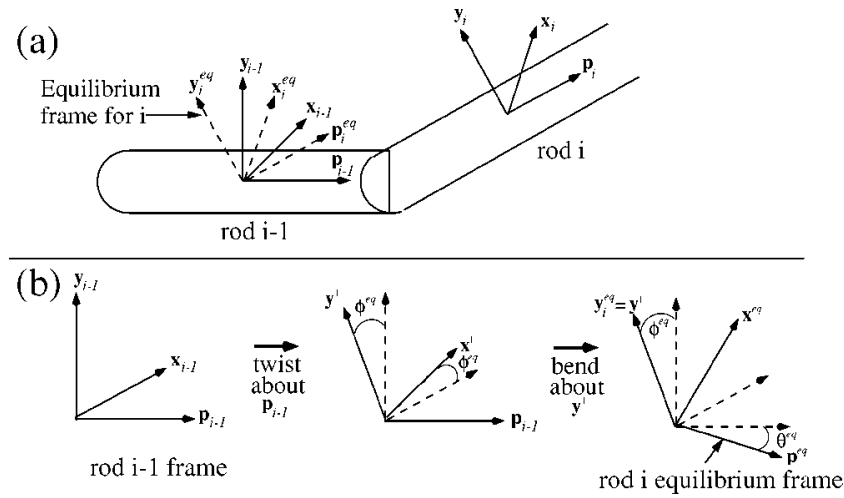

FIG. 2. (a) Frame on $\operatorname{rod} i$, and equilibrium frame for $\operatorname{rod} i$ fixed on $\operatorname{rod} i-1$ used to define fiber equilibrium shape. (b) Relation between rod $i-1$ frame and rod $i$ equilibrium frame.

The angles $\theta^{\mathrm{eq}}$ and $\phi^{\mathrm{eq}}$ are specified to mimic a variety of equilibrium fiber shapes. For an intrinsically straight fiber, $\theta^{\mathrm{eq}}=0$ and $\phi^{\mathrm{eq}}=0$. For a U-shaped fiber whose centerline lies in a plane and has an intrinsic radius of curvature $R_{u}, \theta^{\text {eq }}$ $=2 \tan ^{-1}\left(l / 2 R_{u}\right)$ and $\phi^{\mathrm{eq}}=0\left(\theta^{\mathrm{eq}} \approx l / R_{u}\right.$ for $\left.R_{u} \gg l\right)$. For a helical fiber whose centerline encircles a cylinder of radius $R_{h}$ and rises a distance $H$ in one period, $\cos \theta^{\mathrm{eq}}=\cos \alpha \cos ^{2}(\beta / 2)+\sin ^{2}(\beta / 2)$ and $\cos \phi^{\mathrm{eq}}=\cos \alpha \sin ^{2}(\beta / 2)+\cos ^{2}(\beta / 2)$, where $\alpha=2 \tan ^{-1}\left(l / 2 R_{h}\right)$ and $\beta / 2=\tan ^{-1}\left(H / 4 R_{h}\right)$.

Bending and twisting torques are applied when a chain deforms from its equilibrium shape. The bending torque in hinge $i$ is

$$
\boldsymbol{Y}_{i}^{\text {bend }}=-\kappa_{b} \alpha_{b, i} \boldsymbol{e}_{b, i},
$$

where $\kappa_{b}$ is the bending constant, $\alpha_{b, i}=\cos ^{-1}\left(\boldsymbol{p}_{i} \cdot \boldsymbol{p}_{i}^{\mathrm{eq}}\right)$ is the bending angle, and

$$
\boldsymbol{e}_{b, i}=\left(\frac{\boldsymbol{p}_{i} \times \boldsymbol{p}_{i}^{\mathrm{eq}}}{\left|\boldsymbol{p}_{i} \times \boldsymbol{p}_{i}^{\mathrm{eq}}\right|}\right)
$$

is the bending torque direction. For small deformations, the chain bending stiffness is related to the bending stiffness of an elastic cylinder by $l \kappa_{b} \approx E_{Y} I$ where $E_{Y}$ is the Young's modulus of the fiber material and $I$ is the area moment of inertia, ( $I$ $=\pi R^{4} / 4$ for a circular cylinder with radius $R$ ). The twisting torque in hinge $i$ is

$$
\boldsymbol{Y}_{i}^{\mathrm{twist}}=-\kappa_{t} \alpha_{t, i} c_{i},
$$

where $\kappa_{t}$ is the twisting constant, $\alpha_{t, i}=\cos ^{-1}\left(\hat{y}_{i}^{\perp} \cdot \hat{y}_{i}^{\mathrm{eq}, \perp}\right)$ is the twisting angle, and

$$
\hat{\boldsymbol{y}}_{i}^{\perp}=\frac{\left(\boldsymbol{\delta}-\boldsymbol{c}_{i} \boldsymbol{c}_{i}\right) \cdot \hat{\boldsymbol{y}}_{i}}{\left|\left(\boldsymbol{\delta}-\boldsymbol{c}_{i} \boldsymbol{c}_{i}\right) \cdot \hat{\boldsymbol{y}}_{i}\right|}, \quad \hat{\boldsymbol{y}}_{i}^{\mathrm{eq}, \perp}=\frac{\left(\boldsymbol{\delta}-\boldsymbol{c}_{i} \boldsymbol{c}_{i}\right) \cdot \hat{\boldsymbol{y}}_{i}^{\mathrm{eq}}}{\left|\left(\boldsymbol{\delta}-\boldsymbol{c}_{i} \boldsymbol{c}_{i}\right) \cdot \hat{y}_{i}^{\mathrm{eq}}\right|},
$$

are the projections of vectors $\hat{\boldsymbol{y}}_{i}$ and $\hat{\boldsymbol{y}}_{i}^{\mathrm{eq}}$ perpendicular to unit vector connecting adjacent rod c.m.s, $\boldsymbol{c}_{i}=\left(\boldsymbol{r}_{i}-\boldsymbol{r}_{i-1}\right) /\left|\boldsymbol{r}_{i}-\boldsymbol{r}_{i-1}\right|$. For small deformations, $l \kappa_{t} \approx G J$, where $G$ is the shear modulus of the fiber material and $J$ is the appropriate area moment of inertia $\left(J=\pi R^{4} / 2\right.$ for a circular cylinder with radius $R$ ). In this work, $\kappa_{t} / \kappa_{b}=0.67$, which for an elastic cylinder corresponds to a Poisson's ratio of 0.5. For simple shear flow, the sum of the dimensionless bending and twisting torques in hinge $i$ is 


$$
\boldsymbol{Y}_{i}^{*}=\frac{\boldsymbol{Y}_{i}}{\pi \mu \dot{\gamma} l^{3}}=-\frac{\kappa_{b}}{\pi \mu \dot{\gamma} l^{3}}\left[\alpha_{b, i} \boldsymbol{e}_{b, i}+0.67 \alpha_{t, i} \boldsymbol{c}_{i}\right]
$$

where

$$
\frac{\kappa_{b}}{\pi \mu \dot{\gamma} l^{3}} \equiv \kappa_{b}^{*}=\frac{N^{4}}{\pi}\left(\frac{E_{Y} I}{\mu \dot{\gamma} L^{4}}\right)
$$

is the ratio of the elastic restoring torque scale to hydrodynamic torque scale. The dimensionless fiber stiffness DS $\equiv E_{Y} I /\left(\mu \dot{\gamma} L^{4}\right)$ is related to the bending ratio BR, defined in Eq. (1), by $\mathrm{DS}=\pi \mathrm{BR} /\left[32\left(\ln 2 r_{e}-1.5\right)\right]$ for cylindrical fibers. The elastic energy stored in a fiber is

$$
E_{e}=\frac{E_{Y} I N}{L} \sum_{i=2}^{N}\left(\alpha_{b, i}^{2}+0.67 \alpha_{t, i}^{2}\right) .
$$

In Eqs. (10), (11), and (13), we have assumed that the fiber deformations are small (i.e., $\tan \alpha_{b, i} \approx \alpha_{b, i}$ ). Even for the most flexible fibers investigated here (DS $=7.7$ $\times 10^{-5}$ ), the resulting error in the average bending torque is only $5 \%$.

The orientation of each rod can be represented by four Euler parameters [Wittenburg (1977)] $\boldsymbol{q}_{i}=\left(q_{0}, q_{1}, q_{2}, q_{3}\right)_{i}$, which are related to the unit vectors $\left(\hat{\boldsymbol{x}}_{i}, \hat{\boldsymbol{y}}_{i}, \boldsymbol{p}_{i}\right)$ by

$$
\begin{aligned}
& q_{0}=\frac{1}{2} \sqrt{1+\mathcal{R}_{11}+\mathcal{R}_{22}+\mathcal{R}_{33}}, \quad q_{1}=\frac{1}{2} \frac{\mathcal{R}_{23}-\mathcal{R}_{32}}{\sqrt{1+\mathcal{R}_{11}+\mathcal{R}_{22}+\mathcal{R}_{33}}}, \\
& q_{2}=\frac{1}{2} \frac{\mathcal{R}_{31}-\mathcal{R}_{13}}{\sqrt{1+\mathcal{R}_{11}+\mathcal{R}_{22}+\mathcal{R}_{33}}}, \quad q_{3}=\frac{1}{2} \frac{\mathcal{R}_{12}-\mathcal{R}_{21}}{\sqrt{1+\mathcal{R}_{11}+\mathcal{R}_{22}+\mathcal{R}_{33}}},
\end{aligned}
$$

where $\mathcal{R}_{11(i)}=\hat{\boldsymbol{x}}_{i} \cdot \boldsymbol{e}_{x}, \mathcal{R}_{12(i)}=\hat{\boldsymbol{x}}_{i} \cdot \boldsymbol{e}_{y}, \mathcal{R}_{21(i)}=\hat{\boldsymbol{y}}_{i} \cdot \boldsymbol{e}_{x}$, etc. The orthogonal rotation matrix

$$
\boldsymbol{R}_{i}=\left[\begin{array}{ccc}
q_{0}^{2}+q_{1}^{2}-q_{2}^{2}-q_{3}^{2} & 2\left(q_{1} q_{2}+q_{0} q_{3}\right) & 2\left(q_{1} q_{3}-q_{0} q_{2}\right) \\
2\left(q_{1} q_{2}-q_{0} q_{3}\right) & q_{0}^{2}-q_{1}^{2}+q_{2}^{2}-q_{3}^{2} & 2\left(q_{2} q_{3}+q_{0} q_{1}\right) \\
2\left(q_{1} q_{3}+q_{0} q_{2}\right) & 2\left(q_{2} q_{3}-q_{0} q_{1}\right) & q_{0}^{2}-q_{1}^{2}-q_{2}^{2}+q_{3}^{2}
\end{array}\right]_{i}
$$

(where $\mathcal{R}_{i}^{\dagger}=\mathcal{R}_{i}^{-1}$ ), relates the vector $\hat{\boldsymbol{y}}_{i}$ in the space frame to the body fixed vector $\hat{\boldsymbol{y}}_{i[\text { body } i]}=(0,1,0)$ by $\hat{\boldsymbol{y}}_{i}=\boldsymbol{\mathcal { R }}_{i}^{\dagger} \cdot \hat{\boldsymbol{y}}_{i \text { [bodyi] }}$. Analogous expressions relate $\hat{\boldsymbol{x}}_{i}$ and $\boldsymbol{p}_{i}$ to $\hat{\boldsymbol{x}}_{i[\text { body } i]}=(1,0,0)$ and $\boldsymbol{p}_{i \text { body } i]}=(0,0,1)$. The vectors $\hat{\boldsymbol{y}}_{i}^{\mathrm{eq}}$ and $\boldsymbol{p}_{i}^{\mathrm{eq}}$, needed in twisting and bending torque expressions, respectively, are obtained by

$$
\hat{\boldsymbol{y}}_{i}^{\mathrm{eq}}=\boldsymbol{\mathcal { R }}_{i-1}^{\dagger} \cdot \boldsymbol{\mathcal { R }}_{\mathrm{eq}}^{\dagger} \cdot \hat{\boldsymbol{y}}_{i[\mathrm{eq} i]}^{\mathrm{eq}},
$$

where $\hat{\boldsymbol{y}}_{i \text { [eqi] }}^{\mathrm{eq}}=(0,1,0)$, and an analogous expression for $\boldsymbol{p}_{i}^{\mathrm{eq}}$. The rotation matrix $\boldsymbol{\mathcal { R }}_{\mathrm{eq}}$ is defined as

$$
\boldsymbol{R}_{\mathrm{eq}}=\left[\begin{array}{ccc}
\cos \theta^{\mathrm{eq}} \cos \phi^{\mathrm{eq}} & \cos \theta^{\mathrm{eq}} \sin \phi^{\mathrm{eq}} & -\sin \theta^{\mathrm{eq}} \\
-\sin \phi^{\mathrm{eq}} & \cos \phi^{\mathrm{eq}} & 0 \\
\sin \theta^{\mathrm{eq}} \cos \phi^{\mathrm{eq}} & \sin \theta^{\mathrm{eq}} \sin \phi^{\mathrm{eq}} & \cos \theta^{\mathrm{eq}}
\end{array}\right],
$$


where $\boldsymbol{\mathcal { R }}_{\text {eq }}=\boldsymbol{\mathcal { R }}_{\text {bend }} \cdot \boldsymbol{\mathcal { R }}_{\text {twist }}$ is the product of a bending and twisting rotation, as illustrated in Fig. 2(b).

The fiber contour length is held constant by requiring that $\boldsymbol{r}_{i}+(l / 2) \boldsymbol{p}_{i}=\boldsymbol{r}_{i+1}$ $-(l / 2) \boldsymbol{p}_{i+1}$ for each pair of adjacent rods in a chain. Taking the time derivative leads to the constraint

$$
\dot{\boldsymbol{r}}_{i}-\dot{\boldsymbol{r}}_{i+1}+\frac{l}{2}\left(\boldsymbol{\omega}_{i} \times \boldsymbol{p}_{i}+\boldsymbol{\omega}_{i+1} \times \boldsymbol{p}_{i+1}\right)=\mathbf{0} .
$$

Substituting Eqs. (6) and (9) for the translational and angular velocities yields

$$
\mathcal{Q}_{i}^{*} \cdot \boldsymbol{X}_{i}^{*}+\mathcal{S}_{i}^{*} \cdot \boldsymbol{X}_{i+1}^{*}+\mathcal{T}_{i}^{*} \cdot \boldsymbol{X}_{i+2}^{*}=\mathcal{V}_{i}^{*},
$$

where

$$
\begin{gathered}
\mathcal{Q}_{i}^{*}=\boldsymbol{A}_{i}^{-1 *}-\frac{3}{4 Y^{C}}\left(\boldsymbol{\delta}-\boldsymbol{p}_{i} \boldsymbol{p}_{i}\right), \\
\mathcal{S}_{i}^{*}=-\left(\boldsymbol{A}_{i}^{-1 *}+\boldsymbol{A}_{i+1}^{-1 *}\right)-\frac{3}{4 Y^{C}}\left(2 \boldsymbol{\delta}^{-} \boldsymbol{p}_{i} \boldsymbol{p}_{i}-\boldsymbol{p}_{i+1} \boldsymbol{p}_{i+1}\right), \\
\mathcal{T}_{i}^{*}=\boldsymbol{A}_{i+1}^{-1 *}-\frac{3}{4 Y^{C}}\left(\boldsymbol{\delta}-\boldsymbol{p}_{i+1} \boldsymbol{p}_{i+1}\right), \\
\mathcal{V}_{i}^{*}=-r_{p_{r}}\left(\boldsymbol{p}_{i} \times \boldsymbol{g}_{i}^{*}+\boldsymbol{p}_{i+1} \times \boldsymbol{g}_{i+1}^{*}\right)+\boldsymbol{A}_{i}^{-1 *} \cdot \boldsymbol{F}_{i}^{\mathrm{ext} *}-\boldsymbol{A}_{i+1}^{-1 *} \cdot \boldsymbol{F}_{i+1}^{\mathrm{ext} *}+\boldsymbol{v}_{i}^{\infty *}-\boldsymbol{v}_{i+1}^{\infty *}, \\
\boldsymbol{g}_{i}^{*}=\boldsymbol{\omega}_{i}^{\mathrm{jeff} *}+\frac{3}{4 r_{p_{r}}^{2} Y^{C}} \sum_{j} t_{i j}^{*}-\frac{1}{Y^{C}}\left(\boldsymbol{Y}_{i}^{*}-\boldsymbol{Y}_{i+1}^{*}\right), \\
\boldsymbol{F}_{i}^{\mathrm{ext} *}=\boldsymbol{F}_{i}^{b *}+\sum_{j} \boldsymbol{f}_{i j}^{*} .
\end{gathered}
$$

The $N-1$ inextensibility constraints [Eq. (19) for $1 \leqslant i \leqslant N-1$ ] can be solved simultaneously for the $N-1$ unknown constraint forces $\boldsymbol{X}_{i}^{*}$ in a chain $\left(\boldsymbol{X}_{1}^{*}=\mathbf{0}\right)$, provided all interparticle forces $f_{i j}^{*}$ are known. For a chain of five rods, the system of equations takes the form

$$
\left[\begin{array}{cccc}
\mathcal{S}_{1} & \mathcal{T}_{1} & \mathbf{0} & \mathbf{0} \\
\mathcal{Q}_{2} & \mathcal{S}_{2} & \mathcal{T}_{2} & \mathbf{0} \\
\mathbf{0} & \mathcal{Q}_{3} & \mathcal{S}_{3} & \mathcal{T}_{3} \\
\mathbf{0} & \mathbf{0} & \mathcal{Q}_{4} & \mathcal{S}_{4}
\end{array}\right]^{*} \cdot\left[\begin{array}{l}
\boldsymbol{X}_{2} \\
\boldsymbol{X}_{3} \\
\boldsymbol{X}_{4} \\
\boldsymbol{X}_{5}
\end{array}\right]^{*}=\left[\begin{array}{l}
\mathcal{V}_{1} \\
\mathcal{V}_{2} \\
\mathcal{V}_{3} \\
\mathcal{V}_{4}
\end{array}\right]^{*}
$$

The $(3 N-3) \times(3 N-3)$ matrix is banded, thus computational effort for solving this system of equations can be made to scale linearly with $N$.

Including fiber interactions requires determining the minimum distance between neighboring rod surfaces. The algorithm we employ is summarized in Appendix A.

Repulsive interparticle forces acting normal to fiber surfaces are employed to represent the fibers' excluded volume. Two different types of forces can be employed. The short range position dependent force 


$$
\boldsymbol{f}_{i j}^{\mathrm{rep}}=-F \exp \left[-20\left(g_{i j}-2\right)\right] \boldsymbol{n}_{i j}
$$

can be used, where $g_{i j} \equiv G_{i j} / R$, and $F=120 \pi \mu L R \dot{\gamma}$ is empirically found to eliminate surface overlaps. Here, interparticle forces are applied for $g_{i j} \leqslant 2.33$.

Alternatively, repulsive forces can be applied that satisfy the no-approach constraint

$$
\Delta \boldsymbol{v}_{i j} \cdot \boldsymbol{n}_{i j}=0,
$$

where $\Delta \boldsymbol{v}_{i j}$ is the relative velocity of the rod surfaces at the interaction point

$$
\Delta \boldsymbol{v}_{i j}=\dot{\boldsymbol{r}}_{i}+S_{i j} \boldsymbol{\omega}_{i} \times \boldsymbol{p}_{i}-\dot{\boldsymbol{r}}_{j}-S_{j i} \boldsymbol{\omega}_{j} \times \boldsymbol{p}_{j}+0.5 G_{i j}\left(\boldsymbol{\omega}_{i}+\boldsymbol{\omega}_{j}\right) \times \boldsymbol{n}_{i j} .
$$

Here, forces are applied for $g_{i j} \leqslant 2.01$, simulating mechanical contact between rods. Substituting rod velocities and angular velocities from Eqs. (6) and (9) for $C$ contacts in a cluster of $M$ fibers yields a system of equations for the repulsive force magnitudes $\boldsymbol{f}_{i j}^{\mathrm{rep}} \cdot \boldsymbol{n}_{i j}(<0)$.

Simulations of unconnected rigid rods $\left(N=1, r_{p}=20, n L^{3}=20\right)$ in shear flow with these two methods agree fairly well. Using position dependent repulsive forces results in an average of 0.32 interactions per fiber compared to 0.25 contacts per fiber for repulsive forces that satisfy Eq. (22). Suspension rheological properties agree similarly closely. In results reported here, short range, position dependent repulsive forces are employed, as this method is computationally simpler.

Static friction, which prevents fibers from sliding over one another, is incorporated through no-slip constraints on the motion of interacting particles

$$
\Delta \boldsymbol{v}_{i j} \cdot \boldsymbol{t}_{i j}^{(1)}=0, \quad \Delta \boldsymbol{v}_{i j} \cdot \boldsymbol{t}_{i j}^{(2)}=0 .
$$

Here $\boldsymbol{t}_{i j}^{(1)}$ and $\boldsymbol{t}_{i j}^{(2)}$ are unit vectors tangent to both fiber surfaces at the point of closest approach

$$
\boldsymbol{t}_{i j}^{(1)}=\frac{\left(\boldsymbol{\delta}-\boldsymbol{n}_{i j} \boldsymbol{n}_{i j}\right) \cdot \boldsymbol{e}_{x}}{\left|\left(\boldsymbol{\delta}-\boldsymbol{n}_{i j} \boldsymbol{n}_{i j}\right) \cdot \boldsymbol{e}_{x}\right|}, \quad \boldsymbol{t}_{i j}^{(2)}=\frac{\boldsymbol{n}_{i j} \times t_{i j}^{(1)}}{\left|\boldsymbol{n}_{i j} \times t_{i j}^{(1)}\right|} .
$$

When friction is applied along with position dependent repulsive interparticle forces, the condition

$$
\boldsymbol{f}_{i j}^{\mathrm{fric}} \cdot \boldsymbol{n}_{i j}=0
$$

is imposed on each friction force $f_{i j}^{\text {fric }}$.

Equations (24) and (26) are nondimensionalized, and translational and angular velocities from Eqs. (6) and (9) are substituted, yielding a $3 C \times 3 C$ system of equations for the unknown friction force vectors in a cluster of $M$ fibers connected through $C$ interaction points. These equations contain $3 M(N-1)$ unknown constraint forces $X_{i}^{*}$, and must be solved together with $3 M(N-1)$ inextensibility constraints [Eq. (19)]. We employ an approximate technique, described below.

Friction forces are applied at interaction point $k$ if $\left|f_{k}^{\text {fric }}\right| \leqslant \mu_{f}^{\text {stat }}\left|f_{k}^{\text {rep }}\right|$, where $\mu_{f}^{\text {stat }}$ is the static friction coefficient. If $\left|f_{k}^{\text {fric }}\right|>\mu_{f}^{\text {stat }}\left|f_{k}^{\text {rep }}\right|$ at one or more interaction points, the frictional interaction with the highest value of $\left|f^{\mathrm{fric}}\right| /\left|f^{\mathrm{rep}}\right|$ is removed (a repulsive force is still applied), and friction forces are recalculated. This procedure is repeated until $\left|f_{k}^{\text {fric }}\right|$ $\leqslant \mu_{f}^{\text {stat }}\left|f_{k}^{\text {rep }}\right|$ at all interaction points. This scheme mimics a sliding friction coefficient of zero, similar to the rigid fiber simulations of Thomasset et al. (1997). 


\section{B. Computational details}

In numerical simulations, an approximate technique is adapted wherein constraint forces $\boldsymbol{X}_{i}^{*}$ from the previous time step are used to solve for current friction forces. With this simplification, the motions of adjacent rods in chains are temporarily uncoupled. Friction forces at only $c$ contacts in a cluster of rods (i.e., a group of rods connected through frictional interaction points) must to be calculated simultaneously, rather than $C$ contact forces in a cluster of fibers (typically $c \approx 0.02 C$ ). For a cluster of rods with $c$ interactions, the dimensionless forms of the constraints in Eqs. (24) and (26), combined with rod translational and angular velocities [Eqs. (6) and (9)], yield a $3 c \times 3 c$ system of equations for the friction forces

$$
\left[\begin{array}{cccc}
\boldsymbol{U}_{1} & \boldsymbol{W}_{12} & \cdots & \boldsymbol{W}_{1 c} \\
\boldsymbol{W}_{21} & \boldsymbol{U}_{2} & & \vdots \\
\vdots & & \ddots & \\
\boldsymbol{W}_{c 1} & \cdots & & \boldsymbol{U}_{c}
\end{array}\right] \cdot\left[\begin{array}{c}
\boldsymbol{f}_{1}^{\mathrm{ric} *} \\
\vdots \\
\boldsymbol{f}_{c}^{\mathrm{rric} *}
\end{array}\right]=\left[\begin{array}{c}
\boldsymbol{Z}_{1} \\
\vdots \\
\boldsymbol{Z}_{c}
\end{array}\right] .
$$

Here $\boldsymbol{U}_{k}$ and $\boldsymbol{W}_{k l}$ are second order tensors, each $\boldsymbol{Z}_{k}$ is a vector. These terms are described in Appendix B.

At each simulation time step, bending and twisting torques are first evaluated, followed by a search for interaction points and calculation of position dependent forces. If rods $i$ and $j$ interact, frictional interactions are not considered between $i$ and either $j$ -1 or $j+1$ (rods adjacent to $j$ in its chain). Likewise, $\operatorname{rod} j$ does not interact with $i$ -1 and $i+1$ through frictional forces. This avoids the numerically unstable situation of having two friction forces applied at closely spaced positions near a hinge. Repulsive forces are applied for all $g_{i j} \leqslant 2.33$.

Once all interactions are found, rods are sorted into clusters. The friction forces in each cluster are calculated using Eq. (27) with constraint forces $X_{i}^{*}$ from the previous time step (friction forces are not calculated the first time step). Then, current constraint forces are found by Eq. (19), and rod motion is calculated with Eqs. (6) and (9). Fiber c.m. velocities are calculated and $\boldsymbol{r}_{\text {c.m. }}$ updated by

$$
\boldsymbol{r}_{\text {c.m. }}(\gamma+\Delta \gamma)=\boldsymbol{r}_{\text {c.m. }}(\gamma)+\left[1.5 \dot{\boldsymbol{r}}_{\text {c.m. }}(\gamma)-0.5 \dot{\boldsymbol{r}}_{\mathrm{c} . \mathrm{m} .}(\gamma-\Delta \gamma)\right] \Delta \gamma .
$$

Rod Euler parameters $\boldsymbol{q}_{i}$ are updated by

$$
\left[\begin{array}{l}
\dot{q}_{0} \\
\dot{q}_{1} \\
\dot{q}_{2} \\
\dot{q}_{3}
\end{array}\right]_{i}=\frac{1}{2}\left[\begin{array}{cccc}
0 & -\omega_{b, x} & -\omega_{b, y} & -\omega_{b, z} \\
\omega_{b, x} & 0 & \omega_{b, z} & -\omega_{b, y} \\
\omega_{b, y} & -\omega_{b, z} & 0 & \omega_{b, x} \\
\omega_{b, z} & \omega_{b, y} & -\omega_{b, x} & 0
\end{array}\right]_{i}\left[\begin{array}{l}
q_{0} \\
q_{1} \\
q_{2} \\
q_{3}
\end{array}\right]_{i},
$$

and

$$
\boldsymbol{q}(\gamma+\Delta \gamma)=\boldsymbol{q}(\gamma)+[1.5 \dot{\boldsymbol{q}}(\gamma)-0.5 \dot{\boldsymbol{q}}(\gamma-\Delta \gamma)] \Delta \gamma .
$$

The vector $\boldsymbol{\omega}_{b, i}=\boldsymbol{\mathcal { R }}_{i} \cdot \boldsymbol{\omega}_{i}$ is the rod $i$ angular velocity in the body $i$ frame. Each chain is regrown using Eq. (2) with the new orientations and $\boldsymbol{r}_{\text {c.m. }}$.

Comparisons with an exact technique, in which the motion and forces in each cluster of fibers are calculated simultaneously, show that the approximate technique gives con- 
TABLE I. Dimensionless rotation periods $T \dot{\gamma}$ for model fibers with BR $=2$. ${ }^{1}$ Values $T \dot{\gamma}=2 \pi\left(r_{e}+1 / r_{e}\right)$ with $r_{e}$ from empirical correlation of Cox (1971)].

\begin{tabular}{ccccc}
\hline \hline $\begin{array}{c}\text { Fiber } \\
\text { aspect ratio }\end{array}$ & $\begin{array}{c}\text { Experimental } \\
\text { period } T \dot{\gamma}^{1}\end{array}$ & $\begin{array}{c}\text { Model } \\
\text { fiber }\end{array}$ & $\begin{array}{c}\text { Model fiber } \\
\text { period } T \dot{\gamma}\end{array}$ & $\begin{array}{c}r_{e_{r}} / r_{p_{r}} \\
\text { in simulation }\end{array}$ \\
\hline 50 & 197.1 & $5 \times\left(r_{p_{r}}=10\right)$ & 198.8 & 0.75 \\
$\prime \prime$ & $\prime$ & $10 \times\left(r_{p_{r}}=5\right)$ & 196.8 & 0.78 \\
70 & 264.8 & $5 \times\left(r_{p_{r}}=14\right)$ & 263.1 & 0.70 \\
80 & 297.6 & $8 \times\left(r_{p_{r}}=10\right)$ & 298.4 & 0.71 \\
100 & 363.3 & $10 \times\left(r_{p_{r}}=10\right)$ & 361.5 & 0.69 \\
150 & 522.2 & $5 \times\left(r_{p_{r}}=30\right)$ & 520.9 & 0.63 \\
280 & 919.0 & $14 \times\left(r_{p_{r}}=20\right)$ & 921.8 & 0.61 \\
\hline \hline
\end{tabular}

sistent suspension properties for $\Delta \gamma \leqslant 2 \times 10^{-4}$ [Schmid (1999)]. In the simulations reported here, $\Delta \gamma=2 \times 10^{-4}$.

Explicit, rather than position dependent, repulsive forces can be applied with friction forces. In this case, the no-slip constraints [Eq. (24)] are imposed, along with the noapproach constraint [Eq. (22)]. Comparisons show that the number of close range interactions with position dependent forces is within 5\% of the number of physical contacts with explicit repulsive forces for flexible fiber simulations with $r_{p}=80, N=8$, DS $\equiv E_{Y} I /\left(\mu \dot{\gamma} L^{4}\right)=7.7 \times 10^{-4}, \quad \theta^{\mathrm{eq}}=1.4, \quad \phi^{\mathrm{eq}}=1.1, \quad \mu_{f}^{\text {stat }}=\infty, \quad$ and $n L^{3}=26.2$ [Schmid (1999)]. Thus, we employ position dependent repulsive forces exclusively, and take the number of close interactions to be a good estimate of the number of true mechanical contacts.

Rigid fiber simulations, [Eq. (27) is used with $N=1 ; \boldsymbol{X}_{i}^{*}$ and $\boldsymbol{Y}_{i}^{*}$ on the right hand side set to zero], with $r_{p}=20, \mu_{f}^{\text {stat }}=\infty$, and $n L^{3}=10$ predict consistent steady state suspension properties, and only $5 \%$ slower equilibration as the simulation box side length is decreased from $B=4 L$ to $B=2 L$ [Schmid (1999)]. Here we perform simulations in cubic boxes with side lengths $1.6 L \leqslant B \leqslant 4 L$.

Neglecting fluid and particle inertia suggests that Reynolds numbers are limited to $\operatorname{Re} \equiv \rho \dot{\gamma} L D / \mu \leqslant 0.1$. For typical pulp fibers with length $L=2.5 \mathrm{~mm}$ and diameter $D$ $=32 \mu \mathrm{m} \quad\left[\right.$ Smook (1982)] suspended in water with $\rho=10^{3} \mathrm{~kg} / \mathrm{m}^{3}$ and $\mu$ $=10^{-3} \mathrm{~Pa} \mathrm{~s}$, simulations with $\mathrm{DS}=0.008(\mathrm{BR}=0.25)$ imply that fiber stiffnesses are limited to $E I \leqslant 4 \times 10^{-16} \mathrm{~N} \mathrm{~m}^{2}$. Although such fibers appear stiff in simulations (i.e., they do not bend appreciably; see below), such values for $E I$ are much smaller than experimentally measured values for wood fibers [Kerekes and Tam Doo (1985)]. Simulations with larger DS can be performed at increased computational cost.

\section{SINGLE FIBER AND HOMOGENEOUS SUSPENSION SIMULATIONS}

This model successfully replicates Jeffery orbits of isolated, neutrally buoyant, rigid fibers. The equivalent aspect ratios of the rigid rods $r_{e_{r}}$ are chosen such that the orbit periods of stiff model fibers $(\mathrm{BR}=2)$ match experimentally measured orbit periods ( $T \dot{\gamma}=2 \pi\left(r_{e}+1 / r_{e}\right)$, where $r_{e}$ is related to the fiber aspect ratio $r_{p}$ by the correlation $\left.r_{e}=1.24 r_{p} / \sqrt{\ln r_{p}}[\operatorname{Cox}(1971)]\right)$. Model fiber rotation periods are independent of initial fiber orientation, in agreement with Jeffery's analysis [Jeffery (1922)]. Table I summarizes the equivalent rod aspect ratios used in the simulations. 


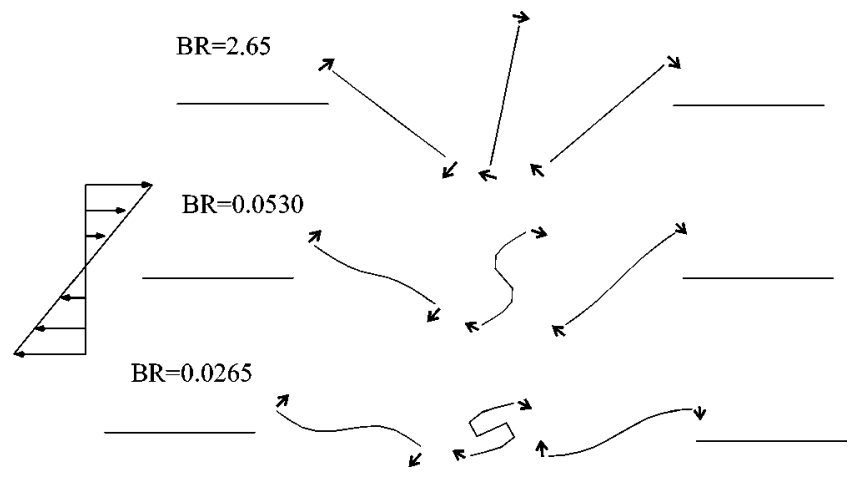

FIG. 3. Snapshots of intrinsically straight, model fibers $\left(r_{p}=50, N=10\right)$ of three different stiffnesses, undergoing $C=\infty$ orbits.

The model reproduces isolated fiber motions in shear flow over a wide range of fiber stiffnesses. Figure 3 shows snapshots of model fibers with three different values of the BR [Eq. (1)] undergoing orbits in the flow/gradient plane (" $C=\infty$ " orbits [Mason (1954)]). The minimum radius of curvature attained by a smooth line that is tangent to each rod at its c.m. (the model fiber "centerline") during a $C=\infty$ orbit is plotted as a function of BR for intrinsically straight model fibers of various aspect ratios in Fig. 4. Intrinsically straight model fibers do not bend appreciably until $B R \leqslant 0.1$; thus they are stiffer than actual fibers, which bend for $B R \leqslant 1$. The symmetrical $S$ shapes of intrinsically straight model fibers also differ qualitatively from the $C$ shapes observed in experiments [Forgacs and Mason (1959b)]. To obtain $C$ shapes during flexible fiber rotations, model fibers are given small permanent deformations (Fig. 5). The bending behavior of model fibers with intrinsic radii of curvature $R_{u}=10 L$, shown by the filled symbols in Fig. 4(a), agrees with qualitative experimental observations of Forgacs and Mason (1959b), represented by the dashed line in that figure. A quantitative comparison with the data of Salinas and Pittman (1981) in Fig. 4(b) demonstrates that small permanent deformations in model fibers are needed to accurately capture correct fiber bending behaviorintrinsically straight fibers act much stiffer than actual fibers.

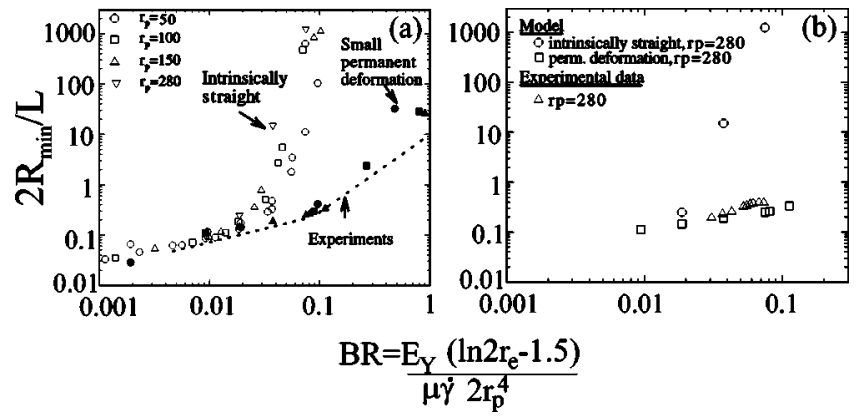

FIG. 4. Dimensionless minimum radius of curvature $R_{\min }$ attained by fibers in $C=\infty$ orbits as a function of BR. (a) Comparison of intrinsically straight and permanently deformed $\left(R_{u}=10 L\right)$ model fibers of $r_{p}$ $=50,100,150$, and 280 with qualitative observations of Forgacs and Mason (1959a). (b) Comparison of intrinsically straight and permanently deformed model fibers of $r_{p}=280$ with measurements of Salinas and Pittman (1981). 


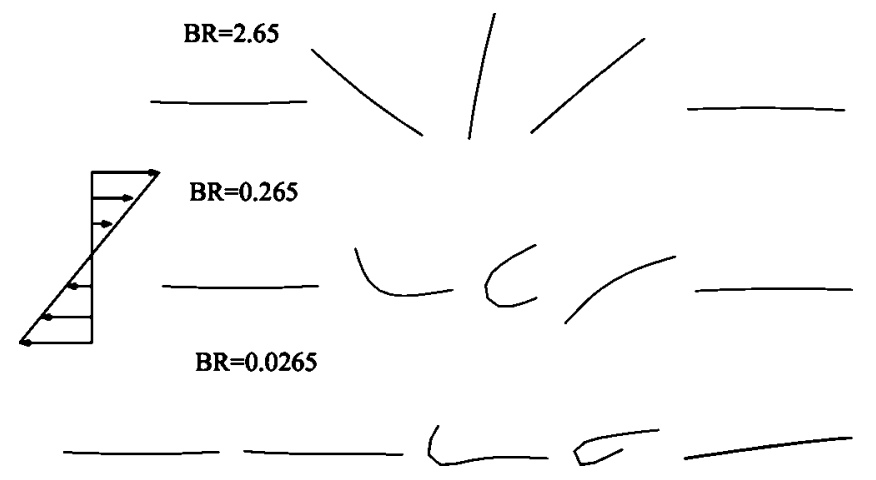

FIG. 5. Snapshots of permanently deformed (intrinsic radius of curvature $R_{u}=10 L$ ), model fibers with $r_{p}$ $=50, N=10$, and three different stiffnesses undergoing $C=\infty$ orbits. For stiff fibers $(\mathrm{BR}=2)$ slightly deformed with $R_{u}=10 L$, the change in the orbit period relative to that of a straight fiber varies roughly linearly with aspect ratio, from a $9 \%$ decrease for $r_{p}=50$ to a $37 \%$ decrease for $r_{p}=150$.

Simulations capture the rheological behavior of homogeneously dispersed, flexible fiber suspensions. The slender-body approximation for the fiber contribution to the deviatoric stress is [Mackaplow and Shaqfeh (1996)]

$$
\begin{aligned}
\boldsymbol{\tau}^{\mathrm{fib}}= & \frac{n}{2}\left\langle\sum_{i=1}^{N} \int_{-l / 2}^{l / 2}[s \boldsymbol{p} \boldsymbol{f}(s)+s \boldsymbol{f}(s) \boldsymbol{p}]_{i} d s_{i}+\left[\boldsymbol{F}_{i}^{h}\left(\boldsymbol{r}_{i}-\boldsymbol{r}_{\mathrm{c} . \mathrm{m} .}\right)+\left(\boldsymbol{r}_{i}-\boldsymbol{r}_{\mathrm{c} . \mathrm{m} .}\right) \boldsymbol{F}_{i}^{h}\right]\right\rangle \\
& +\mathrm{IT}+O\left(\frac{1}{r_{p}}\right),
\end{aligned}
$$

where the second term on the right hand side accounts for the moment exerted by the hydrodynamic force on each rod about its chain c.m. [Batchelor (1970b); Sundararajakumar and Koch (1997)]. Brackets \langle\rangle denote averages over all fibers in the suspension, $s_{i}$ is the position along the rod $i$ axis, and IT is an isotropic term that is not of interest here. The hydrodynamic force per unit length $f\left(s_{i}\right)$ at point $s_{i}$ on rod $i$ is taken as the leading order slender body theory approximation [Batchelor (1970a)],

$$
\boldsymbol{f}\left(s_{i}\right)=3 \pi \mu\left[X_{s b}^{A} \boldsymbol{p}_{i} \boldsymbol{p}_{i}+Y_{s b}^{A}\left(\boldsymbol{\delta}-\boldsymbol{p}_{i} \boldsymbol{p}_{i}\right)\right] \cdot\left(\boldsymbol{v}^{\infty}\left(s_{i}\right)-\dot{\boldsymbol{r}}_{i}-s_{i} \dot{\boldsymbol{p}}_{i}\right),
$$

where $Y_{s b}^{A}=2 X_{s b}^{A}=4 /\left(3 \ln 2 r_{p}\right)$ are scalar resistance functions, $\dot{\boldsymbol{r}}_{i}$ is the $\operatorname{rod} i$ c.m. translational velocity, and $\boldsymbol{v}^{\infty}\left(s_{i}\right)$ is the ambient fluid velocity at position $s_{i}$ on $\operatorname{rod} i$. The hydrodynamic force on rod $i$ is approximated as $\boldsymbol{F}_{i}^{h}=3 \pi \mu l\left[X_{s b}^{A} \boldsymbol{p}_{i} \boldsymbol{p}_{i}+Y_{s b}^{A}\left(\boldsymbol{\delta}-\boldsymbol{p}_{i} \boldsymbol{p}_{i}\right)\right]$ $\cdot\left(\boldsymbol{v}^{\infty}\left(\boldsymbol{r}_{i}\right)-\dot{\boldsymbol{r}}_{i}\right)$ for calculating the stress. For simple shear flow $v_{x}^{\infty}=\dot{\gamma} y$, the dimensionless fiber contribution to the stress is (neglecting IT)

$$
\begin{aligned}
\boldsymbol{\tau}^{\mathrm{fib}} /\left(\frac{\pi \mu \dot{\gamma} n L^{3}}{6 N^{3} \ln 2 r_{p}}\right)= & \left\langle\sum_{i=1}^{N}\left(p_{y} \boldsymbol{p} \boldsymbol{e}_{x}+p_{y} \boldsymbol{e}_{x} \boldsymbol{p}\right)_{i}-\left(\boldsymbol{p} \dot{\boldsymbol{p}}^{*}+\dot{\boldsymbol{p}}^{*} \boldsymbol{p}\right)_{i}-\left(p_{x} p_{y} \boldsymbol{p} \boldsymbol{p}\right)_{i}\right\rangle \\
& +\frac{3}{r_{p_{r}}^{2}}\left\langle\sum_{i=1}^{N}\left[\boldsymbol{\delta}-\frac{1}{2} \boldsymbol{p}_{i} \boldsymbol{p}_{i}\right] \cdot\left(\boldsymbol{v}^{\infty} *\left(\boldsymbol{r}_{i}^{*}\right)-\dot{\boldsymbol{r}}_{i}^{*}\right)\left(\boldsymbol{r}_{i}^{*}-\boldsymbol{r}_{\mathrm{c} . \mathrm{m} .}^{*}\right)\right\rangle .
\end{aligned}
$$




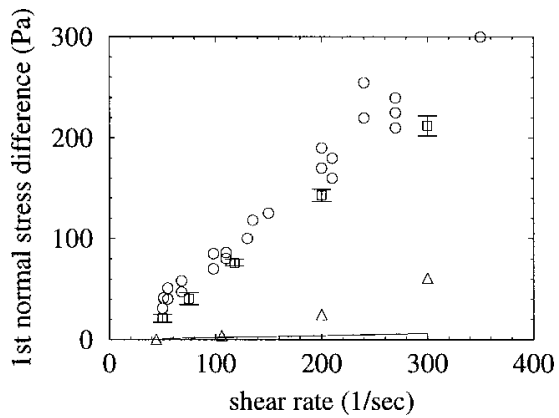

FIG. 6. First normal stress difference as a function of shear rate for $r_{p}=100, \phi_{v}=0.01$ : experimental measurements of Goto et al. (1986) (circles), our simulations with flexible, intrinsically straight fibers (triangles), and flexible, permanently deformed ( $\left.\theta^{\mathrm{eq}}=0.105, \phi^{\mathrm{eq}}=0.524\right)$ fibers (squares), and rigid fiber simulations of Sundararajakumar and Koch (1997) (solid line).

Simulation results demonstrate that rheological properties are strongly impacted by both fiber flexibility and equilibrium shape. In Fig. 6, the first normal stress difference, $N_{1}=\tau_{x x}-\tau_{y y}$, is plotted as a function shear rate for various fiber suspensions with $r_{p}=100$ and $\phi_{v}=0.01\left(n L^{3}=127\right)$ in simple shear flow. The circles are experimental data reported by Goto et al. (1986) for suspensions of nylon fibers $\left(E_{Y} \approx 3\right.$ $\left.\times 10^{9} \mathrm{~Pa}, L=1.4 \mathrm{~mm}, D=14 \mu \mathrm{m}\right)$ in glycerol $(\mu \approx 4 \mathrm{Pas})$. Simulations of straight, rigid fiber suspensions [Sundararajakumar and Koch (1997)] produce much smaller values of $N_{1}$. Our simulations of intrinsically straight, flexible fibers $[N=7$, DS $\left.=0.368 / \dot{\gamma}\left(\mathrm{s}^{-1}\right)\right]$ produce larger values of $N_{1}$ than in rigid fiber simulations at high shear rates $(\dot{\gamma}>100 / \mathrm{s})$, but they are still considerably smaller than the measured values. Simulations of suspensions of flexible fibers with slightly deformed equilibrium shapes $\left[N=7, \theta^{\text {eq }}=0.105, \phi^{\mathrm{eq}}=0.524\right.$ (shape illustrated in Table II) $]$ produce first normal stress differences that nearly match the experimental values (error bars represent the standard deviation over three runs with different initial configurations).

TABLE II. Summary of fiber equilibrium shapes used in simulations of Fig. 15, and in rheological simulations in Sec. III.

\begin{tabular}{c|c|c|c}
\hline \hline$\theta^{\text {eq }}$ & $\phi^{\text {eq }}$ & Side view & Front view \\
\hline 0.9 & 1.0 & 0 \\
0.4 & 0.7 & 0 & 0 \\
0.5 & 0.1 & \\
0.3 & 0 & \\
0.25 & 0 & \\
0.2 & 0 & & \\
0.1 & 0 & & \\
0.105 & 0.524 & & $\gamma$ \\
\hline \hline
\end{tabular}




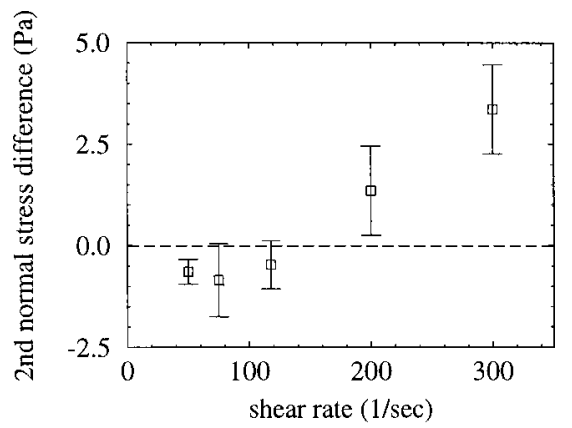

FIG. 7. Second normal stress difference as a function of shear rate for simulations of flexible, permanently deformed fibers with $r_{p}=100, \phi_{v}=0.01, \theta^{\mathrm{eq}}=0.105, \phi^{\mathrm{eq}}=0.524, \mathrm{DS}=0.368 / \dot{\gamma}$.

Nawab and Mason (1958) observed a dramatic Weissenberg rod climbing effect in their experiments with a concentric cylinder rotational viscometer and fibers of $r_{p}$ $>170$ suspended in Castor oil at $\phi_{v} \geqslant 0.002$. They attributed this behavior to the increased rate of fiber flipping caused by fiber flexibility and permanent deformations. For isolated model fibers at $\dot{\gamma}=300 / \mathrm{s}$, the end-to-end vector of a permanently deformed fiber $\left(\theta^{\mathrm{eq}}=0.105, \phi^{\mathrm{eq}}=0.524\right)$ flips through the flow/vorticity plane roughly four times more frequently than for intrinsically straight fibers. In the sheared suspensions studied here, the average permanently deformed fiber holds about 20 times more elastic energy than an intrinsically straight fiber. Hence, in addition to the increased rate of fiber flipping, permanent deformations stimulate elastic fiber deformation, contributing to the elastic character of the fluid.

Second normal stress differences, $N_{2}=\tau_{y y}-\tau_{z z}$, are plotted as a function of shear rate in Fig. 7 for simulations of flexible, permanently deformed fibers $\left(\theta^{\mathrm{eq}}=0.105\right.$, $\left.\phi^{\mathrm{eq}}=0.524\right)$ under the same conditions as above. Simulations predict values that are negative at low shear rates, go through a minimum, and then become positive at higher shear rates.

\section{FLOCCULATION}

Flexible fibers in shear flow often flocculate, generating heterogeneous distributions of fiber mass. Our simulations show that flocculation can be induced by interfiber friction, in the absence of attractive forces between fibers. Below we describe how flocculation behavior depends on the coefficient of friction, fiber stiffness, equilibrium shape, and concentration.

\section{A. Effect of friction coefficient}

Simulations are performed with suspensions of helical, flexible fibers $\left(\theta^{\text {eq }}=0.9\right.$, $\left.\phi^{\mathrm{eq}}=1.0\right)$ with $r_{p}=80, \mathrm{DS} \equiv E_{Y} I /\left(\mu \dot{\gamma} L^{4}\right)=7.7 \times 10^{-3}$, at a volume fraction $\phi_{v}$ $=0.0032\left(n L^{3}=26.2\right)$, and for various friction coefficients $\mu_{f}^{\text {stat }}$. Snapshots of fiber configurations after shearing suspensions to a shear strain $\gamma=2000$ are illustrated in Fig. 8 for $\mu_{f}^{\text {stat }}=5,10$, and $\infty$. For $\mu_{f}^{\text {stat }}=5$, the fibers remain homogeneously dispersed. For $\mu_{f}^{\text {stat }}=10$ and $\infty$, the suspensions exhibit the formation of distinct fiber flocs. This behavior does not change as the simulation box size is increased from $B=2.5 \mathrm{~L}$ to $B$ $=3.5 L$ [Schmid (1999)].

Flocs form in the absence of attractive forces between fibers-friction alone drives flocculation. However, the static friction coefficient required to cause flocculation is 

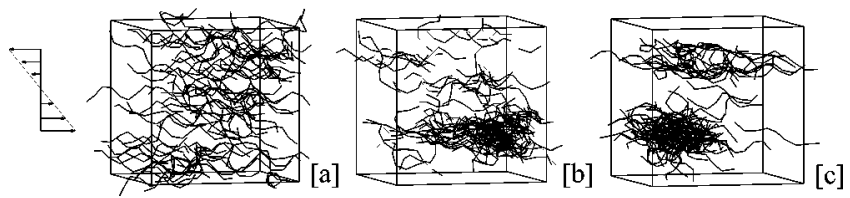

FIG. 8. Suspensions with $r_{p}=80, N=8$, DS $=7.7 \times 10^{-3}, \theta^{\text {eq }}=0.9, \phi^{\text {eq }}=1.0, n L^{3}=26.2$ for: (a) $\mu_{f}^{\text {stat }}=5$, (b) $\mu_{f}^{\text {stat }}=10$, and (c) $\mu_{f}^{\text {stat }}=\infty$, after strains of $\gamma=2000$.

much larger than the experimentally measured value of approximately 0.5 for pulp fiber suspensions [Andersson and Rasmuson (1997); Amelina et al. (1998)]. Possible explanations for this discrepancy are discussed in Sec. V

Flocculation induced by increasing $\mu_{f}^{\text {stat }}$ can be quantified with the fiber c.m. pair distribution function $g(r)$ [Allen and Tildesley (1987)], where $r$ is the distance between c.m.s. Time-averaged pair distribution functions, calculated for $\gamma \geqslant 1000$, are plotted in Fig. 9 for $\mu_{f}^{\text {stat }}=0,5,10$, and $\infty$. For $\mu_{f}^{\text {stat }}=0$ and 5, $g(r)<1$ at small separations. For $\mu_{f}^{\text {stat }}=10$ and $\infty, g(r)>1$ at small separations, reflecting the tendency of fibers to reside in flocs. Again, this aggregation occurs without attractive forces between fibers.

Theories of fiber flocculation attribute the formation of coherent networks to fibers elastic interlocking [Meyer and Wahren (1964), Kerekes et al. (1985), Farnood et al. (1994)]. Fibers are held in networks by friction forces, with the requisite normal forces arising from elastic energy stored in fibers. At least three contacts per fiber are needed to hold a fiber in the network. The average number of contacts per fiber $\left\langle n_{c}\right\rangle$ (averaged over all fibers in the suspension) is plotted as a function of strain in Fig. 10 for the same simulations depicted in Fig. 9. A static friction coefficient of 5 leads to about twice as many contacts per fiber as with purely repulsive interparticle forces $\left(\mu_{f}^{\text {stat }}=0\right)$, but in both cases, $\left\langle n_{c}\right\rangle$ fluctuates about a small value throughout the entire simulation. In contrast, for $\mu_{f}^{\text {stat }}=10$ and $\infty,\left\langle n_{c}\right\rangle$ first increases with strain, and then levels off at large $\gamma$. For these flocculated suspensions, $\left\langle n_{c}\right\rangle>3$, consistent with the mechanical interlocking mechanism of flocculation.

The magnitude of contact forces, and the extent of elastic fiber deformation, are directly linked to friction. As $\mu_{f}^{\text {stat }}$ increases, the average frictional and repulsive interparticle forces, $\left\langle f^{\text {fric } *}\right\rangle \equiv\left\langle\left|f^{\text {fric } *}\right|\right\rangle$ and $\left\langle f^{\text {rep } *}\right\rangle \equiv\left\langle\left|f^{\text {rep } *}\right|\right\rangle$, both increase (Table III). The average elastic energy per fiber $\left\langle E_{e}^{*}\right\rangle$ also increases with $\mu_{f}^{\text {stat }}$. These observations

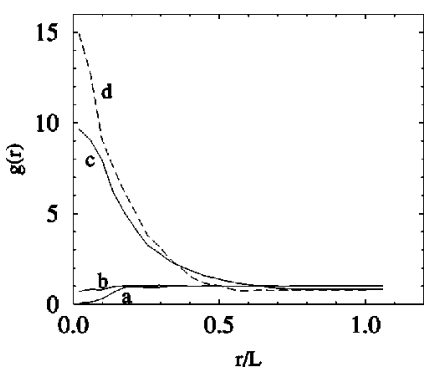

FIG. 9. Fiber c.m. pair distribution functions for sheared suspensions under conditions in Fig. 8: (a) $\mu_{f}^{\text {stat }}$ $=0$, (b) $\mu_{f}^{\text {stat }}=5$, (c) $\mu_{f}^{\text {stat }}=10$, and (d) $\mu_{f}^{\text {stat }}=\infty$, averaged for $\gamma \geqslant 1000$ [fiber c.m.s may coincide, hence $g(0)$ isn't necessarily zero]. 


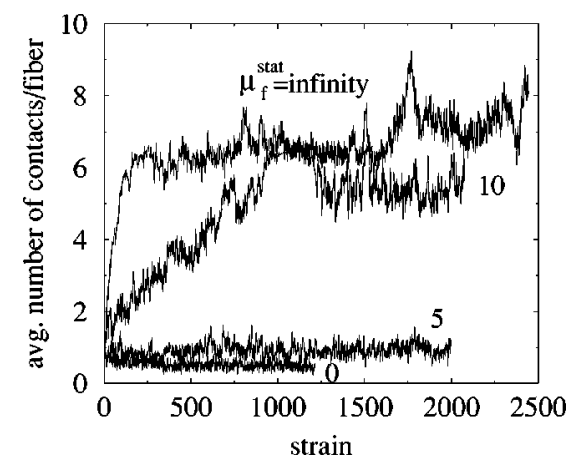

FIG. 10. Average number of contact points per fiber vs strain for sheared supensions with $r_{p}=80, N=8$, DS $=7.7 \times 10^{-3}, n L^{3}=26.2$, and various values of $\mu_{f}^{\text {stat }}$.

demonstrate that interfiber friction not only inhibits contacting fibers from sliding, but also assists in pinning fibers together and deforming them.

Friction also helps store elastic energy in fiber flocs upon the cessation of shear flow. Flocs were formed by shearing a suspension with $r_{p}=70, N=5$, DS $=0.050, \theta^{\text {eq }}$ $=0.8, \phi^{\mathrm{eq}}=0.7, \phi_{v}=0.00125\left(n L^{3}=7.8\right)$, and $\mu_{f}^{\text {stat }}=20$, to a strain of $\gamma$ $=2000$, at which point the average elastic energy per fiber was $E_{e}=0.41 E_{Y} I / L$. The flow was then stopped and the structure allowed to relax, using four different friction coefficients. The fraction of initial elastic energy stored in the fibers is plotted as a function of time for $\mu_{f}^{\text {stat }}=0,10,20$, and 100 in Fig. 11. Here, time is scaled by $t_{s}$ $=\pi \mu L^{4} /\left(62.5 E_{Y} I\right)$, and the dimensionless bending stiffness is DS2 $\equiv E_{Y} I t_{s} /\left(\mu L^{4}\right)$ $=0.050$. For $\mu_{f}^{\text {stat }} \neq 0$, elastic energy is permanently stored in the fibers. This energy storage behavior is consistent with experimental observations showing fiber flocs to retain their structural integrity when shearing is stopped. In experiments of Soszynski and Kerekes (1988), dense nylon fiber flocs are lifted out of the suspending fluid by holding only a few of their constituent fibers.

Friction also helps hold flocs together in unbounded shear flow. Flocs were formed in simulations with shear flow and periodic boundary conditions, $\left(r_{p}=70, N=5\right.$, DS $=0.050, \theta^{\mathrm{eq}}=0.8, \phi^{\mathrm{eq}}=0.7, n L^{3}=7.8$ ), and then removed from the suspension and placed alone in an unbounded shear flow. As fibers break free from the floc, they are carried away by the flow, the number of fibers remaining in a floc (initially containing 80 fibers) is tracked as a function of strain in Fig. 12. With $\mu_{f}^{\text {stat }}=\infty$, the floc loses half of its fibers in a strain of $\gamma \approx 350$, roughly twice as long as with $\mu_{f}^{\text {stat }}=20$, and ten times

TABLE III. Interparticle forces and elastic energy per fiber as a function of $\mu_{f}^{\text {stat }}$ for sheared suspensions with $r_{p}=80, N=8, \mathrm{DS}=7.7 \times 10^{-3}$, $\theta^{\mathrm{eq}}=0.9, \phi^{\mathrm{eq}}=1.0$, and $n L^{3}=26.2$, averaged for $\gamma \geqslant 1000$. Forces are scaled with $3 \pi \mu \dot{\gamma} L R$, and elastic energy with $0.0020 \pi \mu \dot{\gamma} L^{3}$.

\begin{tabular}{rcccc}
\hline \hline$\mu_{f}^{\text {stat }}$ & $\left\langle f^{\text {fric } *}\right\rangle$ & $\left\langle f^{\text {rep } *}\right\rangle$ & $\left\langle f^{\text {fric } *}\right\rangle /\left\langle f^{\text {rep } *}\right\rangle$ & $\left\langle E_{e}^{*}\right\rangle$ \\
\hline 0 & 0 & 0.45 & 0 & 0.23 \\
5 & 1.8 & 1.2 & 1.5 & 0.49 \\
10 & 3.5 & 2.1 & 1.7 & 1.3 \\
$\infty$ & 5.5 & 2.3 & 2.5 & 2.6 \\
\hline \hline
\end{tabular}




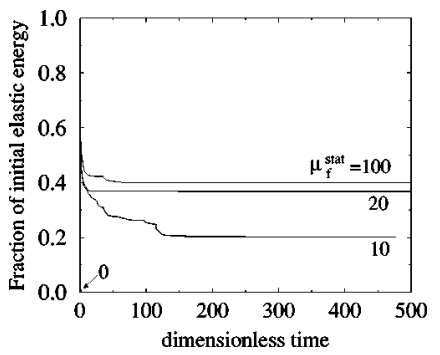

FIG. 11. Fraction of initial elastic energy stored vs $t / t_{s}$ after cessation of shear flow for suspensions with $r_{p}$ $=70, N=5, \mathrm{DS} 2 \equiv E_{Y} I t_{S} /\left(\mu L^{4}\right)=0.050, \theta^{\mathrm{eq}}=0.8, \phi^{\mathrm{eq}}=0.7$, and $n L^{3}=7.8: \mu_{f}^{\text {stat }}=0,10,20$, and 100.

as long as with purely repulsive interfiber forces $\left(\mu_{f}^{\text {stat }}=0\right)$. Thus friction induces fibers to mechanically interlock, and also resists their dispersion.

\section{B. Effect of fiber stiffness}

Fiber stiffness plays an important role in the formation and persistence of simulated flocs. Flocculation in suspensions with $r_{p}=80, N=8, \theta^{\mathrm{eq}}=0.9, \phi^{\mathrm{eq}}=1.0, n L^{3}$ $=26.2$, and $\mu_{f}^{\text {stat }}=\infty$ becomes less prevalent as the dimensionless fiber stiffness decreases, as illustrated by simulation pictures in Fig. 13.

The decreased aggregation is reflected by the fiber c.m. pair distribution function. For $\mathrm{DS}=3.9 \times 10^{-4}$, the shape of $g(r)$ is similar to that for DS $=7.7 \times 10^{-3}$ [curve (d) of Fig. 9], but $g(r=0.01 L)$ drops from 15 to 7. For DS $=7.7 \times 10^{-5}, g(r=0.01 L)$ $\approx 1$.1. Interparticle force magnitudes and elastic energy storage in fibers diminish as fiber stiffness decreases. Table IV presents values of the average frictional and repulsive contact force magnitudes, as well as the average elastic energy per fiber, calculated for $\gamma \geqslant 1000$ for sheared suspensions under the conditions in Fig. 13 and various fiber stiffnesses. Simulations are consistent with the experimental observations that flocs are more prevalent at low shear rates and low suspending fluid viscosities (large dimensionless stiffnesses) [Takeuchi et al. (1983), Zhao and Kerekes (1993)]. Here, the reduction in flocculation is not attributable to fluid and particle inertia effects. Rather, decreased elastic energy storage in fibers relative to hydrodynamic forces produces weaker contact forces, diminishing aggregation.

Kerekes (1995) has suggested that coherent flocs appear in fiber suspensions as the Reynolds number is increased above approximately one. Although our model shows that

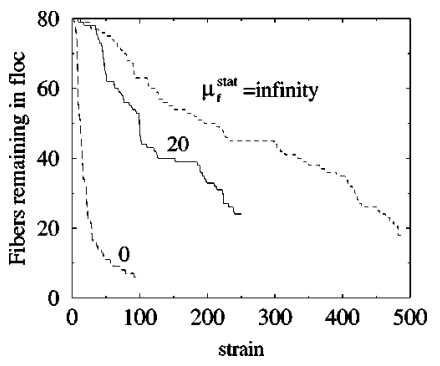

FIG. 12. Fibers remaining in a floc in unbounded shear flow as a function of strain, $r_{p}=70, N=5$, DS $=0.050, \theta^{\mathrm{eq}}=0.8, \phi^{\mathrm{eq}}=0.7: \mu_{f}^{\text {stat }}=0,20$, and $\infty$. 


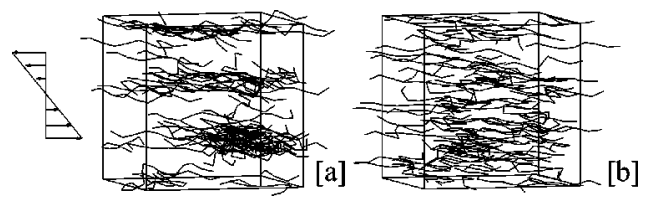

FIG. 13. Sheared suspensions with $r_{p}=80, N=8, \theta^{\mathrm{eq}}=0.9, \phi^{\mathrm{eq}}=1.0, n L^{3}=26.2$ : (a) $\mathrm{DS}=3.9$ $\times 10^{-4}$, and (b) DS $=7.7 \times 10^{-5}$, at $\gamma=1000$ [the suspension with DS $=7.7 \times 10^{-3}$ is illustrated in Fig. $8(\mathrm{c})]$.

flocculation can appear as the viscosity is decreased (i.e., DS is increased), we have neglected particle and fluid inertia, and thus all simulations are performed in the limit of vanishing Reynolds number. Therefore, we cannot comment on Kerekes' suggestion.

Flocs break up more rapidly in unbounded shear flow as fiber stiffness is decreased. A flocculated structure was formed by simulating a suspension $\left(r_{p}=70, N=5\right.$, DS $=0.050, \theta^{\mathrm{eq}}=0.8, \phi^{\mathrm{eq}}=0.7, \mu_{f}^{\text {stat }}=\infty$, and $\left.n L^{3}=7.8\right)$ in shear flow with periodic boundaries to a strain of $\gamma=2000$. A floc containing 80 fibers was then removed and sheared in an unbounded flow with various fiber stiffnesses. The number of fibers remaining in the floc is plotted as a function of strain in Fig. 14, showing that flocs break up more quickly as DS decreases. This result is consistent with experiments of Soszynski and Kerekes (1988), who have found that nylon fiber flocs disperse readily when bending stresses are diminished by heating the flocs above the nylon glass transition temperature. Here, even flocs made of stiff fibers eventually break apart. This supports the dynamic equilibrium hypothesis of Mason and co-workers-fibers constantly enter and leave flocs, and do not irreversibly bind to them [Mason (1950)].

\section{Effect of fiber shape}

Fiber equilibrium shape strongly impacts the bending behavior of single fibers, as well as the first normal stress differences in flexible fiber suspensions (Sec. III). Its dramatic effect on flocculation behavior is now explored. The flocculation behavior of sheared suspensions with $r_{p}=80, N=8$, DS $=7.7 \times 10^{-3}$, and $\mu_{f}^{\text {stat }}=\infty$ is mapped as a function of fiber equilibrium shape and concentration $\left(n L^{3}\right)$ in Fig. 15. Suspensions that remain homogeneously dispersed $[g(r=0.01 L) \leqslant 3]$ to a strain of $\gamma=2000$ are indicated by open symbols; suspensions that flocculate $[g(r=0.01 L)>3]$ within $\gamma$ $=2000$ are indicated by filled symbols. Points are labeled with letters for discussion below.

Suspensions with nearly straight fiber equilibrium shapes at low concentrations remain homogeneously dispersed. At a fixed concentration, increasing permanent fiber deforma-

TABLE IV. Average interparticle forces and elastic energy per fiber as a function of DS for sheared suspensions with $r_{p}=80, N=8, \theta^{\mathrm{eq}}=0.9$, $\phi^{\mathrm{eq}}=1.0, \mu_{f}^{\text {stat }}=\infty$, and $n L^{3}=26.2$, averaged for $\gamma \geqslant 1000$. Forces are scaled with $3 \pi \mu \dot{\gamma} L R$, and elastic energy with $0.0020 \pi \mu \dot{\gamma} L^{3}$.

\begin{tabular}{ccccc}
\hline \hline DS & $\left\langle f^{\text {fric* }}\right\rangle$ & $\left\langle f^{\text {rep } *}\right\rangle$ & $\left\langle f^{\text {fric } *}\right\rangle /\left\langle f^{\text {rep } *}\right\rangle$ & $\left\langle E_{e}^{*}\right\rangle$ \\
\hline $7.7 \times 10^{-3}$ & 5.5 & 2.3 & 2.5 & 2.6 \\
$7.7 \times 10^{-4}$ & 1.8 & 0.68 & 2.7 & 0.90 \\
$3.8 \times 10^{-4}$ & 1.0 & 0.32 & 3.1 & 0.48 \\
$7.7 \times 10^{-5}$ & 0.30 & 0.069 & 4.4 & 0.14 \\
\hline \hline
\end{tabular}




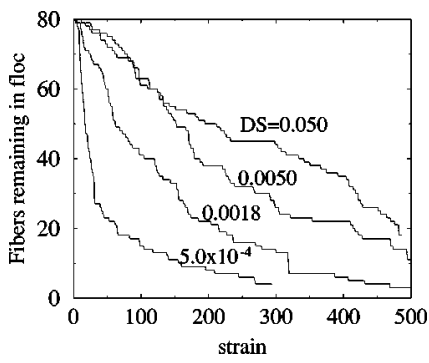

FIG. 14. Fibers remaining in a floc in unbounded shear flow as a function of strain for various fiber stiffnesses, $r_{p}=70, \theta^{\mathrm{eq}}=0.8, \phi^{\mathrm{eq}}=0.7, \mu_{f}^{\text {stat }}=\infty$.

tion (quantified as $\theta^{\mathrm{eq}}+\phi^{\mathrm{eq}}$ ) induces a dramatic transition in suspension structure. For example, U-shaped fibers with $\theta^{\mathrm{eq}}=0.2$ at $n L^{3}=26.2$ remain homogeneously dispersed over a strain of 2000, while fibers with $\theta^{\text {eq }}=0.3$ flocculate, as illustrated in Fig. 16.

Fiber equilibrium shape strongly impacts suspension microstructural and mechanical properties. In Fig. 17(a), $\left\langle n_{c}\right\rangle$ is plotted as a function of strain for suspensions $\mathrm{C}, \mathrm{J}, \mathrm{K}$, and $\mathrm{L}$, illustrating significantly more fiber interactions and faster aggregation for suspension $\mathrm{C}\left(\theta^{\mathrm{eq}}=0.9, \phi^{\mathrm{eq}}=1.0\right)$ than for suspension $\mathrm{L}\left(\theta^{\mathrm{eq}}=0.2, \phi^{\mathrm{eq}}=0\right)$. Highly irregular fiber equilibrium shapes also stimulate elastic fiber deformation in flowing suspensions, and lead to stronger interparticle forces, as exhibited by the values of $\left\langle f^{\text {fric } *}\right\rangle$, $\left\langle f^{\mathrm{rep} *}\right\rangle$, and $\left\langle E_{e}^{*}\right\rangle$ (averaged for $\gamma \geqslant 1000$ ) presented in Table V.

Although irregular fiber equilibrium shapes facilitate aggregation in the presence of friction forces, our simulations do not exhibit flocculation with purely repulsive interparticle forces. Suspensions of stiff ( $\left.\mathrm{DS}=7.7 \times 10^{-3}\right)$ permanently deformed $\left(\theta^{\text {eq }}\right.$ $\left.=0.8, \phi^{\mathrm{eq}}=0.2\right)$ fibers of $r_{p}=80$ with $\mu_{f}^{\text {stat }}=0$ remain homogeneously dispersed even at $n L^{3}=65.5$

\section{Effect of fiber concentration}

Simulations show that flocculation becomes more prevalent with increasing $n L^{3}$, in agreement with theory and experiment [Mason (1954), Kerekes and Schell (1992)]. For sheared suspensions with $r_{p}=80, N=8, \mathrm{DS}=7.7 \times 10^{-3}, \theta^{\mathrm{eq}}=0.5, \phi^{\mathrm{eq}}=0.1$, and $\mu_{f}^{\text {stat }}=\infty$, a distinct transition from homogeneously dispersed to flocculated occurs

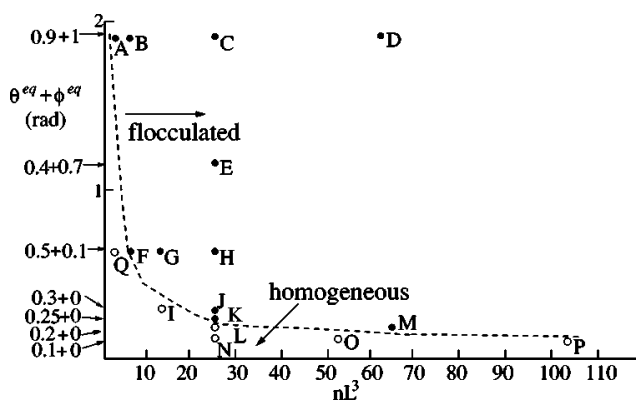

FIG. 15. Flocculation behavior as a function of fiber equilibrium shape and concentration $\left(n L^{3}\right)$ for suspensions with $r_{p}=80, N=8$, DS $=7.7 \times 10^{-3}$, and $\mu_{f}^{\text {stat }}=\infty$. 


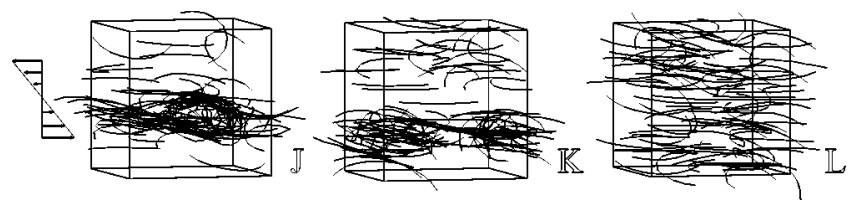

FIG. 16. Suspensions of U-shaped fibers with $r_{p}=80, N=8, \mathrm{DS}=7.7 \times 10^{-3}$, and $\mu_{f}^{\text {stat }}=\infty$ : $\theta^{\text {eq }}$ $=0.3(\mathrm{~J}), \theta^{\mathrm{eq}}=0.25(\mathrm{~K})$, and $\theta^{\mathrm{eq}}=0.2(\mathrm{~L})$ at $\gamma=2000$.

between $n L^{3}=3$ and $n L^{3}=12$, as illustrated in Fig. 18. Suspensions flocculate below $n L^{3}=3$ with $\theta^{\mathrm{eq}}=0.9, \phi^{\mathrm{eq}}=1.0$. In contrast, nearly intrinsically straight fibers $\left(\theta^{\text {eq }}=0.1, \phi^{\text {eq }}=0\right)$ remain uniformly dispersed even for $n L^{3}=105$, as shown in Fig. 19.

Although flocs do not form in simulations of nearly intrinsically straight fibers [for $\theta^{\mathrm{eq}}=0.1, \phi^{\mathrm{eq}}=0, n L^{3}=105, g(r=0.01 L)=1.9$ at $\left.\gamma=2000\right]$, evidence suggests that a transition from a homogeneous suspension to a uniform fiber network occurs between $n L^{3}=52.5$ and $n L^{3}=105$. Upon cessation of shear flow at $n L^{3}=105$, fibers initially holding (on average) $E_{e}=1.1 E_{Y} I / L$ relax to a static configuration by $t$ $=2.7 \mu L^{4} /\left(E_{Y} I\right)$, retaining roughly $40 \%$ of their initial elastic energy. In contrast, at $n L^{3}=52.5$ the elastic energy relaxes to zero by $t=0.2 \mu L^{4} /\left(E_{Y} I\right)$. Kerekes and coworkers [Kerekes and Schell (1992); Kerekes (1995)] also observed a transition to floc formation at sufficiently large concentrations. They found that coherent fiber flocs formed for $n L^{3} \geq 115\left(N_{c} \geq 60\right)$, albeit for different flow fields.

In sheared suspensions with $r_{p}=80, N=8, \mathrm{DS}=7.7 \times 10^{-3}, \theta^{\text {eq }}=0.1, \phi^{\text {eq }}$ $=0$, and $\mu_{f}^{\text {stat }}=\infty$, the average number of contact points per fiber increases from $\left\langle n_{c}\right\rangle=1.7$ at $n L^{3}=52.5$ to $\left\langle n_{c}\right\rangle=4.8$ at $n L^{3}=105$. Upon relaxation of shear flow, $\left\langle n_{c}\right\rangle$ decays to zero for the suspension at $n L^{3}=52.5$, where it increases to $\left\langle n_{c}\right\rangle$ $=5.4$ for the static fiber network at $n L^{3}=105$. Meyer and Wahren (1964) predict $\left\langle n_{c}\right\rangle$ for static networks of straight, randomly oriented fibers. For $r_{p} \gg 1$, Kerekes and Schell (1992) simplify the result to

$$
n L^{3} \approx \frac{8\left\langle n_{c}\right\rangle^{3}}{\left\langle n_{c}\right\rangle-1} .
$$

In Fig. 20, $\left\langle n_{c}\right\rangle$ is plotted as a function of $n L^{3}$ for various sheared and static simulated suspensions. These results show that the simulated structures differ markedly from that

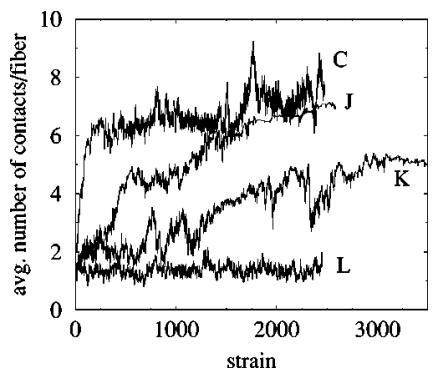

FIG. 17. Average number of contacts per fiber versus strain for sheared suspensions with $r_{p}=80, N=8$, DS $=7.7 \times 10^{-3}, \mu_{f}^{\text {stat }}=\infty$, and $n L^{3}=26.2: \quad \theta^{\text {eq }}=0.9, \quad \phi^{\text {eq }}=1(\mathrm{C}), \theta^{\text {eq }}=0.3, \quad \phi^{\text {eq }}=0(\mathrm{~J}), \theta^{\text {eq }}$ $=0.25, \phi^{\mathrm{eq}}=0(\mathrm{~K}), \theta^{\mathrm{eq}}=0.2, \phi^{\mathrm{eq}}=0(\mathrm{~L})$. 
TABLE V. Average interparticle forces and elastic energy per fiber as a function of equilibrium shape for sheared suspensions with $r_{p}=80, N$ $=8$, DS $=7.7 \times 10^{-3}, \mu_{f}^{\text {stat }}=\infty$, and $n L^{3}=26.2$, averaged for $\gamma$ $\geqslant 1000$. Forces are scaled with $3 \pi \mu \dot{\gamma} L R$, and elastic energy with $0.0020 \pi \mu \dot{\gamma} L^{3}$.

\begin{tabular}{ccccc}
\hline \hline$\theta^{\mathrm{eq}}, \phi^{\mathrm{eq}}(\mathrm{rad})$ & $\left\langle f^{\text {fric } *}\right\rangle$ & $\left\langle f^{\text {rep } *}\right\rangle$ & $\left\langle f^{\text {fric } *}\right\rangle /\left\langle f^{\text {rep } *}\right\rangle$ & $\left\langle E_{e}^{*}\right\rangle$ \\
\hline $0.2,0$ & 2.0 & 0.45 & 4.4 & 0.35 \\
$0.25,0$ & 4.4 & 1.0 & 4.4 & 0.85 \\
$0.3,0$ & 5.7 & 1.6 & 3.6 & 1.5 \\
$0.9,1$ & 5.5 & 2.3 & 2.5 & 2.6 \\
\hline \hline
\end{tabular}

predicted for randomly oriented fibers [Eq. (34); solid curve in Fig. 20], and depends on the interfiber friction coefficient, the equilibrium fiber shape, as well as the imposed flow field.

The average contact force in static, elastically interlocked fiber suspensions with $r_{p}$ $=80, N=8, \mathrm{DS} 2=7.7 \times 10^{-3}$, and $\mu_{f}^{\text {stat }}=\infty$ is impacted by fiber equilibrium shape. For a network with nearly straight fibers $\left(\theta^{\text {eq }}=0.1, \phi^{\text {eq }}=0\right)$ at $n L^{3}=105$, we find $\left\langle f^{\text {fric }}\right\rangle=1400 E_{Y} I R /\left(L^{3}\right)$ and $\left\langle f^{\text {rep }}\right\rangle=550 E_{Y} I R /\left(L^{3}\right)$. For softwood fibers of $L$ $=2.5 \mathrm{~mm}, R=16 \mu \mathrm{m}$, and $E_{Y} I=8 \times 10^{-12} \mathrm{~N} \mathrm{~m}^{2}$, the corresponding average repulsive interparticle force is $\left\langle f^{\mathrm{rep}}\right\rangle=4.5 \mu \mathrm{N}$. In contrast, for a static, flocculated suspension with permanently deformed fibers $\left(\theta^{\mathrm{eq}}=0.9, \phi^{\mathrm{eq}}=1.0\right)$ at a lower concentration $\left(n L^{3}=65.6\right)$, the average contact force magnitudes are larger: $\left\langle f^{\text {fric }}\right\rangle$ $=3520 E_{Y} I R / L^{3}$ and $\left\langle f^{\mathrm{rep}}\right\rangle=1770 E_{Y} I R / L^{3}=14.4 \mu \mathrm{N}$ for softwood fibers. Wahren (1980) estimates

$$
\left\langle f^{\mathrm{rep}}\right\rangle \approx \frac{E_{Y} I\left\langle n_{c}\right\rangle}{L^{2}}
$$

for the average repulsive contact force in an elastically interlocked fiber network. For $n L^{3}=105$, Eq. (34) yields $\left\langle n_{c}\right\rangle \approx 3$, and the average repulsive contact force is predicted to be $\left\langle f^{\text {rep }}\right\rangle=3.8 \mu \mathrm{N}$, in close agreement with our simulations of nearly intrinsically straight fibers. For $n L^{3}=65.6$, Wahren's theory estimates $\left\langle f^{\text {rep }}\right\rangle=2.8 \mu \mathrm{N}$, which is five times lower than our simulations of highly permanently deformed fibers.

\section{CONCLUSION}

Flocs form in particle level simulations in the absence of attractive forces between fibers-interfiber friction and repulsive interactions alone induce flocculation. The behavior and properties of these flocs suggest that they form through an elastic interlocking mechanism; aggregation is more prevalent as elastic energy held in the fibers increases, and flocs contain high percentages of fibers with three or more contact points, remain
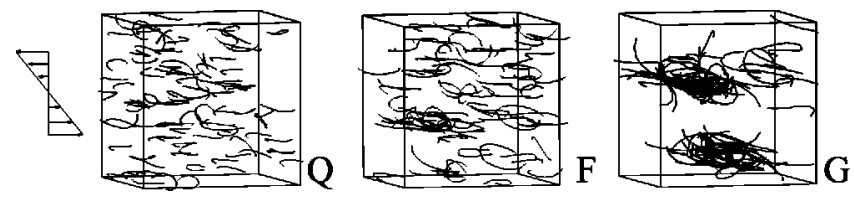

FIG. 18. Sheared suspensions with $r_{p}=80, N=8$, DS $=7.7 \times 10^{-3}, \theta^{\text {eq }}=0.5, \phi^{\text {eq }}=0.1$, and $\mu_{f}^{\text {stat }}$ $=\infty$ for three concentrations: $n L^{3}=3(\mathrm{Q}), n L^{3}=6(\mathrm{~F})$, and $n L^{3}=12(\mathrm{G})$ at $\gamma=1000$. 


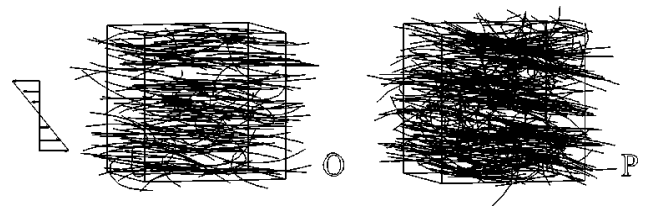

FIG. 19. Sheared suspensions with $r_{p}=80, N=8$, DS $=7.7 \times 10^{-3}, \theta^{\text {eq }}=0.1, \phi^{\text {eq }}=0$, and $\mu_{f}^{\text {stat }}=\infty$ for two concentrations: $n L^{3}=52.5(\mathrm{O})$, and $n L^{3}=105(\mathrm{P})$, at $\gamma=1000$.

coherent upon removal of shear flow, and retain structural integrity when sheared in unbounded flow. High interfiber static friction coefficients, stiff fibers, and large permanent fiber deformations all stimulate increased elastic energy storage in fibers, as well as stronger frictional and repulsive interfiber forces. The friction coefficient, as expected, impacts frictional forces more, while fiber stiffness and equilibrium shape exert more influence on repulsive forces. These simulations have demonstrated that fiber features and interaction forces strongly affect flocculation behavior and floc properties. This technique provides a means of exploring effects that are difficult to incorporate into theories that assume particular suspension structures.

The simulation technique has shortcomings and limitations that should be mentioned. Static friction coefficients that lead to flocculation in simulations are much higher than the experimentally measured value of 0.5 for pulp fiber surfaces [Andersson and Rasmuson (1997), Amelina et al. (1998)]. Possible explanations for this discrepancy include neglecting inter- and intrafiber hydrodynamic interactions, the lack of sliding friction, neglecting attractive interfiber forces, and assuming isotropic fiber bending. The existing techniques for studying long range hydrodynamic interactions between fibers mentioned in the introduction could be implemented, at an increased computational cost. For a typical simulation of 200 fibers, each with $r_{p}=80, N=8, \mathrm{DS}=7.7 \times 10^{-3}$ at $n L^{3}$ $=50$, roughly $60 \mathrm{hrs}$. of computation time is required to simulate a strain of 1000 on a $533 \mathrm{MHz}$ workstation with 256 megabytes of RAM. To probe larger systems, extensive computer speed and memory are required. Future work should also further explore the dependence of flocculation behavior on system parameters, as well as the sensitivity of results to initial conditions.

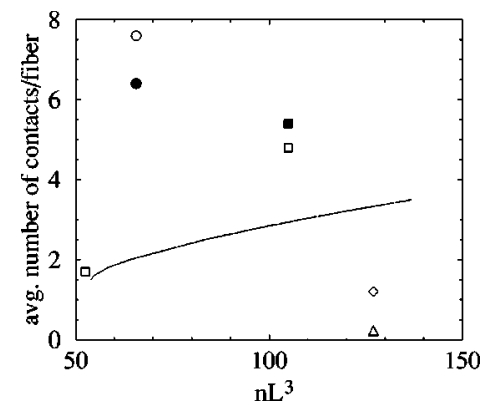

FIG. 20. Comparison of the average number of contact points per fiber $\left\langle n_{c}\right\rangle$ vs $n L^{3}$ : sheared suspensions with $\left(r_{p}, N, \mathrm{DS}, \theta^{\mathrm{eq}}, \phi^{\mathrm{eq}}, \mu_{f}^{\text {stat }}\right)=(80,8,0.0077,0.9,1.0, \infty)$ (open circle), $(80,8,0.0077,0.1,0, \infty)$ (open squares), $(100,7,0.0033,0.105,0.524,0)$ (diamond), and $(100,7,0.0033,0,0,0)$ (triangle); static networks with $(80,8$, $0.0077,0.9,1.0, \infty)$ (filled circle), $(80,6,0.0077,0.1,0, \infty)$ (filled square); Eq. (34) (solid line). 


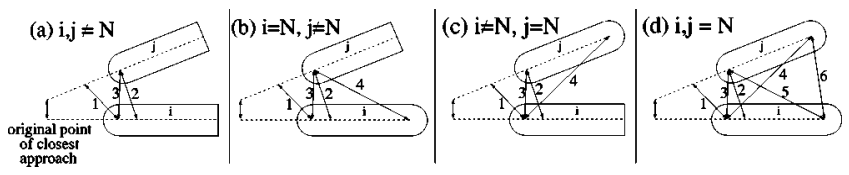

FIG. 21. Four cases for end/side and end/end interactions: separation distances considered in finding the point of closest approach.

\section{ACKNOWLEDGMENTS}

The authors would like to thank Stefan Zauscher for helpful discussions. This work was supported by a Samuel C. Johnson Distinguished Fellowship, and by the National Science Foundation (Grant No. CTS-9502276).

\section{APPENDIX A}

The smallest distance between rods may be between two rod side surfaces, two rod ends, or one side and one end. Consider first the distance between a point on the centerline of $\operatorname{rod} i$ and a point on the centerline of $\operatorname{rod} j, G=\left|\boldsymbol{r}_{i}+x \boldsymbol{p}_{i}-\boldsymbol{r}_{j}-y \boldsymbol{p}_{j}\right|$, where $x$ and $y$ are the distances from the c.m.s of rods $i$ and $j$ to the respective points along their centerlines. The minimum distance between the centerlines is $G_{i j}=\mid \boldsymbol{r}_{i}+S_{i j} \boldsymbol{p}_{i}-\boldsymbol{r}_{j}$ $-S_{j i} \boldsymbol{p}_{j} \mid$, where

$$
S_{i j} \equiv x_{\min }=\frac{\left[\left(\boldsymbol{r}_{i}-\boldsymbol{r}_{j}\right) \cdot \boldsymbol{p}_{j}\right]\left(\boldsymbol{p}_{i} \cdot \boldsymbol{p}_{j}\right)-\left(\boldsymbol{r}_{i}-\boldsymbol{r}_{j}\right) \cdot \boldsymbol{p}_{i}}{1-\left(\boldsymbol{p}_{i} \cdot \boldsymbol{p}_{j}\right)^{2}},
$$

and

$$
S_{j i} \equiv y_{\min }=\frac{\left[\left(\boldsymbol{r}_{j}-\boldsymbol{r}_{i}\right) \cdot \boldsymbol{p}_{i}\right]\left(\boldsymbol{p}_{i} \cdot \boldsymbol{p}_{j}\right)-\left(\boldsymbol{r}_{j}-\boldsymbol{r}_{i}\right) \cdot \boldsymbol{p}_{j}}{1-\left(\boldsymbol{p}_{i} \cdot \boldsymbol{p}_{j}\right)^{2}}
$$

are distances along the centerlines corresponding to the minimum distance between the centerlines. If rods $i$ and $j$ are exactly parallel, which is improbable in suspension simulations, they do not interact.

If $G_{i j}$ is greater than a cutoff distance $G_{c}$ (values of $G_{c}$ for different types of interactions are given in the text), rods $i$ and $j$ do not interact. If $G_{i j}<G_{c}$, and $-l / 2$ $\leqslant S_{i j}, S_{j i} \leqslant l / 2$, then a side/side interaction is considered.

If $G_{i j}<G_{c}$, but $\left|S_{i j}\right|>l / 2$ and/or $\left|S_{j i}\right|>l / 2$, then one of several end/end or end/side interactions occurs. The distances that must be evaluated depend on whether either $\operatorname{rod} i$ or $j$ is the $N$ th $\operatorname{rod}$ in its chain (which has two hemispherical caps). The situations that must be considered are illustrated schematically in Fig. 21.

If $i, j \neq N$, then three distances are compared: (1) the minimum distance from the centerline of rod $i$ at $x=-l / 2$ (the center of curvature of the hemispherical cap) to the centerline of $\operatorname{rod} j ;(2)$ the minimum distance from the centerline of $\operatorname{rod} j$ at $y=-l / 2$ to the centerline of rod $i$; and (3) the distance between the points $x=-l / 2$ on the centerline of $\operatorname{rod} i$ and $y=-l / 2$ on the centerline of $\operatorname{rod} j$. Distance (1) occurs at

$$
y_{\min } \equiv S_{j i}=\left(\boldsymbol{r}_{i}-\frac{l}{2} \boldsymbol{p}_{i}-\boldsymbol{r}_{j}\right) \cdot \boldsymbol{p}_{j}
$$


on the rod $j$ centerline. Likewise, if one or both of $i$ and $j$ are $N$ th rods in a chain, distances are compared as shown in Fig. 21. The shortest distance for which $-l / 2$ $\leqslant S_{i j}, S_{j i} \leqslant l / 2$ is the closest approach between rods $G_{i j}$; if it is less than $G_{c}$, then a $\mathrm{rod} / \mathrm{rod}$ interaction is counted.

\section{APPENDIX B}

Referring to Eq. (27), we denote $s_{i j} \equiv S_{i j} / R$, and suppress rod subscripts $i$ and $j$ on $\boldsymbol{f}_{k}^{\mathrm{fric} *}, \boldsymbol{t}_{k}^{(1)}, \boldsymbol{t}_{k}^{(2)}, \boldsymbol{n}_{k}$, and $g_{k}$ for clarity. Each interaction $k$ between rods $i$ and $j$ ( $i$ $<j$ ) contributes a diagonal term

$$
\boldsymbol{U}_{k}=\left[\begin{array}{ccc}
\leftarrow & {\left[\mathcal{U}_{k}^{\dagger} \cdot t_{k}^{(1)}\right]^{\dagger}} & \rightarrow \\
\leftarrow & {\left[\mathcal{U}_{k}^{\dagger} \cdot t_{k}^{(2)}\right]^{\dagger}} & \rightarrow \\
\leftarrow & n_{k}^{\dagger} & \rightarrow
\end{array}\right],
$$

where the notation $\leftarrow \boldsymbol{v}^{\dagger} \rightarrow$ signifies a vector $\boldsymbol{v}$ placed in a row with $v_{x}$ in column one, $v_{y}$ in column two, and $v_{z}$ in column three. The second order tensor $\mathcal{U}_{k}$ is

$$
\begin{aligned}
\mathcal{U}_{k}= & u_{1} \boldsymbol{\delta}+u_{2} \boldsymbol{p}_{i} \boldsymbol{p}_{i}+u_{3} \boldsymbol{p}_{j} \boldsymbol{p}_{j}+u_{4}\left(\boldsymbol{p}_{i} \boldsymbol{n}_{k}+\boldsymbol{n}_{k} \boldsymbol{p}_{i}\right)+u_{5}\left(\boldsymbol{p}_{j} \boldsymbol{n}_{k}+\boldsymbol{n}_{k} \boldsymbol{p}_{j}\right) \\
& +u_{6}\left(\boldsymbol{\delta}-\boldsymbol{n}_{k} \boldsymbol{n}_{k}\right)-u_{7}\left(\boldsymbol{N}_{k} \cdot\left(\boldsymbol{p}_{i} \boldsymbol{p}_{i}+\boldsymbol{p}_{j} \boldsymbol{p}_{j}\right) \cdot \boldsymbol{N}_{k}\right),
\end{aligned}
$$

where

$$
\begin{aligned}
& u_{1}=\frac{2}{Y^{A}}+\frac{3}{4 r_{p_{r}}^{2} Y^{C}}\left[s_{i j}^{2}+s_{j i}^{2}+g_{k}\left(s_{i j} \boldsymbol{n}_{k} \cdot \boldsymbol{p}_{i}-s_{j i} \boldsymbol{n}_{k} \cdot \boldsymbol{p}_{j}\right)\right], \\
& u_{2}=\left(\frac{1}{X^{A}}-\frac{1}{Y^{A}}\right)-\frac{3 s_{i j}^{2}}{4 r_{p_{r}}^{2} Y^{C}}, \\
& u_{3}=\left(\frac{1}{X^{A}}-\frac{1}{Y^{A}}\right)-\frac{3 s_{j i}^{2}}{4 r_{p_{r}}^{2} Y^{C}}, \quad u_{4}=-\frac{3 s_{i j} g_{k}}{8 r_{p_{r}}^{2} Y^{C}}, \\
& u_{5}=\frac{3 s_{j i} g_{k}}{8 r_{p_{r}}^{2} Y^{C}}, \quad u_{6}=\frac{3 g_{k}^{2}}{16 r_{p_{r}}^{2} Y^{C}}, \\
& u_{7}=\frac{3 g_{k}^{2}}{16 r_{p_{r}}^{2}}\left(\frac{1}{X^{C}}-\frac{1}{Y^{C}}\right), \\
& N_{k}=\left[\begin{array}{ccc}
0 & -n_{k_{z}} & n_{k_{y}} \\
n_{k_{z}} & 0 & -n_{k_{x}} \\
-n_{k_{y}} & n_{k_{x}} & 0
\end{array}\right] .
\end{aligned}
$$

The right hand side vector $\boldsymbol{Z}_{k}$ for each interaction $k$ is 


$$
\boldsymbol{Z}_{k}=\left[\begin{array}{c}
\mathcal{Z}_{k} \cdot \boldsymbol{t}_{k}^{(1)} \\
\mathcal{Z}_{k} \cdot \boldsymbol{t}_{k}^{(2)} \\
0
\end{array}\right]
$$

where

$$
\mathcal{Z}_{k}=\boldsymbol{a}_{j}-\boldsymbol{a}_{i}+s_{i j} \boldsymbol{p}_{i} \times \boldsymbol{b}_{i}-s_{j i} \boldsymbol{p}_{j} \times \boldsymbol{b}_{j}+0.5 g_{k} \boldsymbol{n}_{k} \times\left(\boldsymbol{b}_{i}+\boldsymbol{b}_{j}\right),
$$

and

$$
\begin{gathered}
\boldsymbol{a}_{i}=\boldsymbol{v}_{i}^{\infty *}+\boldsymbol{A}_{i}^{-1 *} \cdot\left(\sum_{m} f_{i m}^{\mathrm{net} *}-\boldsymbol{X}_{i}^{*}+\boldsymbol{X}_{i+1}^{*}+\boldsymbol{F}_{i}^{b *}\right) \\
\boldsymbol{b}_{i}=\boldsymbol{\omega}_{i}^{\mathrm{jeff} *}+\frac{3}{4 r_{p_{r}}^{2} Y^{C}} \boldsymbol{p}_{i} \times\left(r_{p_{r}}\left(\boldsymbol{X}_{i}^{*}+\boldsymbol{X}_{i+1}^{*}\right)+\sum_{m} s_{i m} f_{i m}^{\mathrm{net} *}\right)+\boldsymbol{C}_{i}^{-1 *} \cdot\left(-\boldsymbol{Y}_{i}^{*}+\boldsymbol{Y}_{i+1}^{*}\right) .
\end{gathered}
$$

Here $f_{i m}^{\text {net } *}$ is the net position-dependent force between rods $i$ and $m$, and all $X_{i}^{*}$ are previous time step values. For simulations in simple shear flow and periodic boundary conditions, the term $\boldsymbol{v}_{j}^{\infty *}-\boldsymbol{v}_{i}^{\infty} *=\left(r_{j_{y}}^{*}-r_{i_{y}}^{*}\right) \boldsymbol{e}_{x}$ in $\mathcal{Z}_{k}$ must be adjusted if rod $i$ interacts with a minimum image of rod $j$ from either above or below the main simulation box. In this case, we replace $r_{j_{y}}^{*}-r_{i_{y}}^{*}$ with $r_{j_{y}}^{*}-r_{i_{y}}^{*}-\operatorname{NINT}\left[\left(r_{j_{y}}^{*}-r_{i_{y}}^{*}\right) / B^{*}\right] B^{*}$, where $B^{*}$ $=B / R$ and $\operatorname{NINT}(a / b)$ is the nearest integer to $a / b$.

The off-diagonal tensors

$$
\boldsymbol{W}_{k l}=\left[\begin{array}{ccc}
\leftarrow & {\left[\mathcal{W}_{k l}^{\dagger} \cdot \boldsymbol{t}_{k}^{(1)}\right]^{\dagger}} & \rightarrow \\
\leftarrow & {\left[\mathcal{W}_{k l}^{\dagger} \cdot \boldsymbol{t}_{k}^{(2)}\right]^{\dagger}} & \rightarrow \\
0 & 0 & 0
\end{array}\right]
$$

are nonzero when interaction points $k$ and $l$ occur on the same rod, [the tensor $\boldsymbol{W}_{l k}$ is obtained by exchanging subscripts $k$ and $l$ in Eq. (45)]. Tensors $\mathcal{W}_{k l}$ assume one of four different forms, depending on the indices of the interacting rods. For rods $i$ and $j$ ( $i$ $<j$ ) interacting at point $k$, and rods $i$ and $h(i<h)$ interacting at point $l, \mathcal{W}_{k l}$ $=\mathcal{W}_{k l}^{(1)}$ where

$$
\mathcal{W}_{k l}^{(1)}=w_{1} \boldsymbol{\delta}+w_{2} \boldsymbol{p}_{i} \boldsymbol{p}_{i}+w_{3} \boldsymbol{p}_{i} \boldsymbol{n}_{k}+w_{4} \boldsymbol{n}_{l} \boldsymbol{p}_{i}+w_{5} \boldsymbol{n}_{l} \boldsymbol{n}_{k}-w_{6}\left(\boldsymbol{N}_{k} \cdot \boldsymbol{p}_{i} \boldsymbol{p}_{i} \cdot \boldsymbol{N}_{l}\right),
$$

and

$$
\begin{gathered}
w_{1}=\frac{1}{Y^{A}}+\frac{3}{4 r_{p_{r}}^{2} Y^{C}}\left(s_{i j} s_{i h}+0.5 s_{i j} g_{l} \boldsymbol{n}_{l} \cdot \boldsymbol{p}_{i}+0.5 s_{i h} g_{k} \boldsymbol{n}_{k} \cdot \boldsymbol{p}_{i}+0.25 g_{k} g_{l} \boldsymbol{n}_{k} \cdot \boldsymbol{n}_{l}\right), \\
w_{2}=\left(\frac{1}{X^{A}}-\frac{1}{Y^{A}}\right)-\frac{3 s_{i j} s_{i h}}{4 r_{p_{r}}^{2} Y^{C}}, \quad w_{3}=-\frac{3 s_{i h} g_{k}}{8 r_{p_{r}}^{2} Y^{C}}, \\
w_{4}=-\frac{3 s_{i j} g_{l}}{8 r_{p_{r}}^{2} Y^{C}}, \quad w_{5}=-\frac{3 g_{k} g_{l}}{16 r_{p_{r}}^{2} Y^{C}},
\end{gathered}
$$




$$
w_{6}=-\frac{3 g_{k} g_{l}}{16 r_{p_{r}}^{2}}\left(\frac{1}{X^{C}}-\frac{1}{Y^{C}}\right) .
$$

The tensor $\mathcal{W}_{l k}^{(1)}$ is obtained by exchanging indices $k$ and $l$, and exchanging $s_{i h}$ and $s_{i j}$ everywhere in Eqs. (46) and (47).

If the same rods interact, but $h<i, \mathcal{W}_{k l}=\mathcal{W}_{k l}^{(2)}$ is obtained by first taking $(-1) \times$ Eq. (46), and then replacing $\boldsymbol{n}_{l}$ with $-\boldsymbol{n}_{l}$ everywhere in Eqs. (46) and (47). The tensor $\mathcal{W}_{l k}^{(2)}$ is obtained by exchanging indices $k$ and $l$, and replacing $s_{i j}$ with $-s_{i h}$, and $s_{i h}$ with $-s_{i j}$, everywhere in the expression for $\mathcal{W}_{k l}^{(2)}$.

For rods $i$ and $j(i<j)$ interacting at point $k$, and rods $j$ and $h(j<h)$ interacting at point $l, \mathcal{W}_{k l}=\mathcal{W}_{k l}^{(3)}$ where

$$
\mathcal{W}_{k l}^{(3)}=w_{1} \boldsymbol{\delta}+w_{2} \boldsymbol{p}_{j} \boldsymbol{p}_{j}+w_{3} \boldsymbol{p}_{j} \boldsymbol{n}_{k}+w_{4} \boldsymbol{n}_{l} \boldsymbol{p}_{j}+w_{5} \boldsymbol{n}_{l} \boldsymbol{n}_{k}-w_{6}\left(\boldsymbol{N}_{k} \cdot \boldsymbol{p}_{j} \boldsymbol{p}_{j} \cdot \boldsymbol{N}_{l}\right),
$$

and

$$
\begin{gathered}
w_{1}=-\frac{1}{Y^{A}}+\frac{3}{4 r_{p_{r}}^{2} Y^{C}}\left(-s_{j i} s_{j h}-0.5 s_{j i} g_{l} \boldsymbol{n}_{l} \cdot \boldsymbol{p}_{j}+0.5 s_{j h} g_{k} \boldsymbol{n}_{k} \cdot \boldsymbol{p}_{j}+0.25 g_{k} g_{l} \boldsymbol{n}_{k} \cdot \boldsymbol{n}_{l}\right), \\
w_{2}=-\left(\frac{1}{X^{A}}-\frac{1}{Y^{A}}\right)+\frac{3 s_{j i} s_{j h}}{4 r_{p_{r}}^{2} Y^{C}}, \quad w_{3}=-\frac{3 s_{j h} g_{k}}{8 r_{p_{r}}^{2} Y^{C}}, \\
w_{4}=\frac{3 s_{j i} g_{l}}{8 r_{p_{r}}^{2} Y^{C}}, \quad w_{5}=-\frac{3 g_{k} g_{l}}{16 r_{p_{r}}^{2} Y^{C}}, \\
w_{6}=-\frac{3 g_{k} g_{l}}{16 r_{p_{r}}^{2}}\left(\frac{1}{X^{C}}-\frac{1}{Y^{C}}\right) .
\end{gathered}
$$

The tensor $\mathcal{W}_{l k}^{(3)}$ is obtained by exchanging indices $k$ and $l$, and replacing $s_{j i}$ with $-s_{h j}$, and $s_{h j}$ with $-s_{j i}$ everywhere in Eqs. (48) and (49).

If the same rods interact, but $h<j$, the tensor $\mathcal{W}_{k l}=\mathcal{W}_{k l}^{(4)}$ is obtained by first taking $(-1) \times$ Eq. (48), and then replacing $\boldsymbol{n}_{l}$ with $-\boldsymbol{n}_{l}$ everywhere in Eqs. (48) and (49). The tensor $\mathcal{W}_{l k}^{(4)}$ is then found by exchanging indices $k$ and $l$, and exchanging $s_{j i}$ and $s_{i h}$ everywhere in the expression for $\mathcal{W}_{k l}^{(4)}$.

\section{References}

Allen, M. P. and D. J., Tildesley, Computer Simulation of Liquids (Clarendon, Oxford, 1987).

Amelina, E. A., E. D. Shchukin, A. M. Parfenova, A. I. Bessonov, and I. V. Videnskii, “Adhesion of cellulose fibers in liquid media: 1. Measurement of the contact friction force," Colloid J. USSR 60, 537-540 (1998). Andersson, S. R. and A. Rasmuson, "Dry and wet friction of single pulp and synthetic fibres," J. Pulp Pap. Sci. 23, J5-J11 (1997)

Batchelor, G. K., "Slender-body theory for particles of arbitrary cross-section in Stokes flow," J. Fluid Mech. 44, 419-440 (1970a).

Batchelor, G. K., "The stress system in a suspension of force-free particles," J. Fluid Mech. 41, 545-570 (1970b).

Bennington, C. P. J., R. J. Kerekes, and J. R. Grace, “The yield stress of fiber suspensions," Can. J. Chem. Eng. 68, 748-756 (1990) 
Bretherton, F. P., "The motion of rigid particles in a shear flow at low Reynolds number," J. Fluid Mech. 14, 284-304 (1962).

Claeys, I. L. and J. F. Brady, "Suspensions of prolate spheroids in Stokes flow. 1. Dynamics of a finite number of particles in an unbounded fluid," J. Fluid Mech. 68, 411-442 (1993).

Cox, R. G., "'The motion of long slender bodies in a viscous fluid. 2. Shear flow,'” J. Fluid Mech. 45, 625-657 (1971).

Fan, X. J., N. Phan-Thien, and R. Zheng, “A direct simulation of fiber suspensions," J. Non-Newtonian Fluid Mech. 74, 113-135 (1998).

Farnood, R. R., S. R. Loewen, and C. T. J. Dodson, “Estimation of intra-floc forces,'” APPITA J. 47, 391-396 (1994).

Forgacs, O. L. and S. G. Mason, "Particle motions in sheared suspensions. 9. Spin and deformation of threadlike particles," J. Colloid Interface Sci. 14, 457-472 (1959a).

Forgacs, O. L. S. G. Mason, "Particle motions in sheared suspensions. 10. Orbits of flexible threadlike particles," J. Colloid Interface Sci. 14, 473-491 (1959b).

Goldsmith, H. L. and S. G. Mason, "The microrheology of dispersions," Rheology, edited by F. Eirich (Academic, Oxford, 1967), Vol. 4, pp. 85-201.

Goto, S., H. Nagazono, and H. Kato, "The flow behavior of fiber suspensions in Newtonian fluids and polymer solutions," Rheol. Acta 25, 119-129 (1986).

Harlen, O. G., R. R. Sundararajakumar, and D. L. Koch, "Numerical simulations of a sphere settling through a suspension of neutrally buoyant fibres,"' J. Fluid Mech. 388, 355-388 (1999).

Jeffery, G. B., “The motion of ellipsoidal particles immersed in a viscous fluid," Proc. R. Soc. London, Ser. A 102, 161-179 (1922).

Kerekes, R. J., "Perspectives on fibre flocculation in papermaking," in Proceedings of the International Paper Physics Conference, Niagara-on-the-Lake, Ontario, Sept., 1995 (Canadian Pulp and Paper Association, Montreal, 1995), pp. 23-31.

Kerekes, R. J., R. M. Soszynski, and P. A. Tam Doo, “The flocculation of pulp fibers," in Transactions of the 8th Fundamental Research Symposium, Oxford, England, Sept. 1985, edited by V. Punton (Mechanical Engineering, London, 1985), pp. 265-309.

Kerekes, R. J. and P. A. Tam Doo, "Wet fiber flexibility of some major softwood species pulped by various processes,'” J. Pulp Pap. Sci. 11, J60-J61 (1985).

Kerekes, R. J. and C. J. Schell, "Characterization of fiber flocculation regimes by a crowding factor,' J. Pulp Pap. Sci. 18, J32-J38 (1992).

Kim, S. and S. J. Karrila, Microhydrodynamics-Principles and Selected Applications (ButterworthHeinemann, Boston, 1991).

Mackaplow, M. B. and E. S. G. Shaqfeh, "A numerical study of the rheological properties of suspensions of rigid, non-Brownian fibers," J. Fluid Mech. 329, 155-186 (1996).

Mason, S. G., "The flocculation of pulp suspensions and the formation of paper," Tappi J. 33, 440-444 (1950).

Mason, S. G., "Fiber motions and flocculation," Pulp Pap. Mag. Can. 37, 494-501 (1954).

Meyer, R. and D. Wahren, "On the elastic properties of three-dimensional fibre networks,' Sven. Papperstidn. 67, 432-437 (1964).

Nawab, M. A. and S. G. Mason, “The viscosity of dilute suspensions of threadlike particles," J. Phys. Chem. 62, 1248-1253 (1958).

Ross, R. F. and D. J. Klingenberg, "'Dynamic simulation of flexible fibers composed of linked rigid bodies,'” J. Chem. Phys. 106, 2949-2960 (1997).

Salinas, A. and J. F. T. Pittman, "Bending and breaking fibers in sheared suspensions," Polym. Eng. Sci. 21, 23-31 (1981).

Schmid, C. F., "Simulations of flocculation in flowing fiber suspensions," Ph.D. thesis, University of Wisconsin-Madison, 1999.

Skjetne, P., R. F. Ross, and D. J. Klingenberg, "Simulation of single fiber dynamics," J. Chem. Phys. 107, 2108-2121 (1997).

Smook, G. A., Handbook for Pulp and Paper Technologists (Tappi, Atlanta, GA, 1982).

Soszynski, R. M. and R. J. Kerekes, "Elastic interlocking of nylon fibers suspended in liquid," Nordic Pulp Pap. Res. J. 3, 172-184 (1988).

Sundararajakumar, R. R. and D. L. Koch, "Structure and properties of sheared fiber suspensions with mechanical contacts," J. Non-Newtonian Fluid Mech. 73, 205-239 (1997).

Takeuchi, N., S. Senda, K. Namba, and G. Kuwabara, "Formation and destruction of fiber flocs in a flowing pulp suspension,” APPITA J., 37, 223-230 (1983).

Thomasset, J., M. Grmela and P. J. Carreau, "Microstructure and rheology of polymer melts reinforced by long glass fibres: direct simulations," J. Non-Newtonian Fluid Mech. 73, 195-203 (1997).

Wahren, D., "Fiber network structures in papermaking operations," in Proceedings of the Institute of Paper Chemistry Symposium 'Paper Science and Technology-The Cutting Edge', Appleton, WI, 1979 (Institute of Paper Chemistry, Appleton, WI, 1980), pp. 112-129.

Wittenburg, I.J., Dynamics of Systems of Rigid Bodies (B. G. Teubner, Stuttgart, 1977). 
Yamamoto, S. and T. Matsuoka, "Dynamic simulation of microstructure and rheology of fiber suspensions," Polym. Eng. Sci. 36, 2396-2403 (1996).

Yamane, Y., Y. Kaneda, and M. Doi, “Numerical simulation of semi-dilute suspensions of rodlike particles in shear flow," J. Non-Newtonian Fluid Mech. 54, 405-421 (1994).

Zauscher, S., C. T. Scott, J. L. Willett, and D. J. Klingenberg, "Pulp extrusion for recycling waste papers and paper mill sludges,"' Tappi J. (in press, 2000).

Zhao, R. H. and R. J. Kerekes, "The effect of suspending liquid viscosity on fiber flocculation,” Tappi J. 76, 183-188 (1993). 
